\title{
Selective inhibition of histone deacetylase 1 and 3 improves motor phenotype and alleviates transcriptional dysregulation in Huntington's disease mice
}

Katharina Hecklau ${ }^{1,2}$, Susanne Mueller ${ }^{2,3,5}$, Stefan Paul Koch ${ }^{3,5}$, Mustafa Hussain Mehkary ${ }^{1,2}$, Busra Kilic $^{1,2}$, Christoph Harms ${ }^{2,3,4}$, Philipp Boehm-Sturm ${ }^{2,3,5}$, and Ferah Yildirim ${ }^{1,2,4}$

${ }^{1}$ Department of Neuropsychiatry, Department of Psychiatry and Psychotherapy, ${ }^{2}$ NeuroCure Cluster of Excellence, ${ }^{3}$ Department of Experimental Neurology, Center for Stroke Research Berlin (CSB), ${ }^{4}$ Einstein Center for Neurosciences Berlin, ${ }^{5}$ Charité Core Facility 7T Experimental MRIs, Charité Universitätsmedizin Berlin, Charitéplatz 1, 10117 Berlin, Germany

\section{Corresponding Author:}

Ferah Yildirim

ferah.yildirim@charite.de

Key words:

HDAC inhibition, RGFP109, transcriptional dysregulation, Huntington's disease, R6/1 mouse model, volumetric MRI 


\begin{abstract}
Huntington's disease (HD) is an autosomal dominant neurodegenerative disease characterized by a late clinical onset of psychiatric, cognitive, and motor symptoms. Transcriptional dysregulation is an early and central disease mechanism which is accompanied by epigenetic alterations in HD. Previous studies demonstrated that targeting transcriptional changes by inhibition of histone deacetylases (HDACs), especially the class I HDACs, provides therapeutic effects. Yet, their exact mechanisms of action and the features of HD pathology, on which these inhibitors act remain to be elucidated. Here, using transcriptional profiling, we found that selective inhibition of HDAC1 and HDAC3 by RGFP109 repaired the expression of a number of genes, including the transcription factor genes Neurod 2 and $\mathrm{Nr} 4 \mathrm{a} 2$, and $43 \%$ of the gene sets that were dysregulated by mutant Huntingtin expression in the striatum and improved motor skill learning deficit in the R6/1 mouse model of HD. RGFP109-treated R6/1 mice showed improved coordination on the RotaRod over four consecutive trials, while vehicle-treated R6/1 animals displayed no improvement in coordination skills and fell 50 seconds earlier off the rod in the fourth trial. We also found, by volumetric MRI, a widespread brain atrophy in the R6/1 mice at the symptomatic disease stage, on which RGFP109 showed a modest effect. Collectively, our combined work presents new evidence for specific HDAC1 and HDAC3 inhibition as a therapeutic strategy for alleviating the phenotypic and molecular features of HD.
\end{abstract}




\section{Introduction}

Huntington's disease (HD), an autosomal dominant neurodegenerative disease caused by CAG repeat expansions in the exon I of the Huntingtin (HTT) gene (The Huntington's Disease Collaborative Research Group, 1993), is characterized by progressive psychiatric, motor, and cognitive symptoms and is fatal. In HTT exon 1, normal individuals have 7-34 CAG repeats, while HD patients display more than 40 and in juvenile cases even more than 100 CAG repeats (Duyao et al., 1993). Currently, no disease-modifying treatment is available. Mutant $H T T$ gene causes brain region-specific neuronal dysfunction and degeneration that is most prominent in the striatum during early disease stages and spreads to other brain regions when the disease progresses (Tabrizi et al., 2012).

Transcriptional dysregulation is an early and central pathogenic mechanism in Huntington's disease which has been demonstrated in cell and animal models of HD as well as in human HD brain (Cha, 2000; Hodges et al., 2006; Seredenina and Luthi-Carter, 2012). Transcriptional repression of many genes coding for neurotransmitters, neurotrophins, and their receptors is a hallmark of HD, while genes that are part of stress-response pathways were shown to be upregulated in HD. Some of the key neuronal genes that are consistently reported to be repressed across HD patients and animal models include brain-derived neurotrophic factor $(B d n f)$, preproenkephalin (Penk), dopamine receptor 2 $(\operatorname{Drd} 2)$, and dopamine receptor 1a ( $\operatorname{rrd} 1)$. Accordingly, it has been shown that overexpression of $B d n f$ and Penk is neuroprotective and improves disease outcome in HD (Bissonnette et al., 2013; Zuccato et al., 2005; Zuccato et al., 2011). Furthermore, dopamine agonist and antagonists play an important role in symptomatic treatment of HD patients (Cepeda et al., 2014; Coppen and Roos, 2017; Rangel-Barajas et al., 2015). However, the mechanisms driving transcriptional dysregulation in HD are not fully understood. It was suggested that mutant HTT directly interacts with transcription factors and DNA (Benn et al., 2008). On the other hand there is strong evidence for epigenetic mechanisms such as histone acetylation, histone ubiquitination, histone trimethylation (H3K9me3, H3K4me3), and DNA methylation contributing to selective changes in gene expression in HD pathogenesis (Bett et al., 2009; Ferrante et al., 2004; Hervas-Corpion et al., 2018; Ryu et al., 2006; Seo et al., 2008; Vashishtha et al., 2013).

Histone hyperacetylation by histone acetyltransferases (HATs) is generally associated with gene expression and histone hypoacetylation by histone deacetylases (HDACs) with gene repression (Kurdistani et al., 2004). Global as well as gene specific histone hypoacetylation at promoters of down-regulated genes were shown in various HD models (Sadri-Vakili et al., 2007; Yildirim et al., 2019). Mutant HTT leads to hypoacetylation by directly binding HATs such as CBP, reducing their activity and resulting in gene repression (Cong et al., 2005; Jiang et al., 2006; Nucifora et al., 2001; Steffan et al., 2000). Overexpressing CBP reverses these effects and leads to decreased mutant HTT toxicity (Jiang et al., 2006; Steffan et al., 2000). Similar neuroprotective effects are achieved by 
inhibiting the opponent, HDACs. HDAC inhibition has been shown to ameliorate transcriptional changes in HD and improve behavioral deficits across different experimental models (Butler and Bates, 2006; Ferrante et al., 2003; Ferrante et al., 2004; Hockly et al., 2003; Kazantsev and Thompson, 2008; Pallos et al., 2008; Ryu et al., 2006; Steffan et al., 2001). Beyond HD, such chromatin targeting strategies exert therapeutic effects in other neurological conditions such as ischemic injury, Alzheimer disease, and Amyotrophic lateral sclerosis as previously reported by us and others (Faraco et al., 2006; Harrison and Dexter, 2013; Meisel et al., 2006; Ryu et al., 2005; Schweizer et al., 2015; Yang et al., 2017; Yildirim et al., 2008). These findings provide significant support for the disease-modifying therapeutic potential of epigenome-targeting strategies such as inhibition of histone deacetylation.

Initially, HDAC inhibitors have been studied and applied in cancer research identifying non-selective HDAC inhibitors such as Trichostatin A (TSA) and Vorinostat as anti-cancer drugs. In recent years, however, selective HDAC inhibitors have been developed (Wagner et al., 2015) and there is growing evidence that especially HDAC class I inhibitors are effective in suppressing pathogenic mechanisms in mouse models of HD (Chen et al., 2013; Chiu et al., 2011; Jia et al., 2012; Jia et al., 2016; Lim et al., 2011; Suelves et al., 2017; Thomas et al., 2008). RGFP109 is a HDAC class I inhibitor selective for HDAC1 and HDAC3. RGFP109 confirmed safety in a recent Phase I clinical trial in patients of Friedreich Ataxia Syndrome and enhanced the mRNA levels of the key disease gene frataxin (FXN) in patient blood mononuclear cells indicating its potentials for disease amelioration (Soragni et al., 2014). In another study, RGFP109 treatment led to significantly decreased levels of L-dopa induced dyskinesia in a Parkinson's disease marmosets model (Johnston et al., 2013). A short-term treatment of HD R6/2 mice with RGFP109 modified the expression levels of 4 out of 13 measured diseaseassociated genes in the striatum (Jia et al., 2012). So far, a comprehensive investigation of the effects of RGFP109 on different aspects of HD, such as behavioral disease phenotypes or brain atrophy, has not been carried out.

In the present study, we tested the therapeutic effects of the selective HDAC inhibitor RGFP109 in the R6/1 mouse model of HD. The outcome of HDAC1 and HDAC3 inhibition was assessed by a set of behavioral tests and genome-wide transcriptional analysis of the striatum. Moreover, we performed volumetric magnetic resonance imaging (MRI) of the brain in living animals. Our results demonstrate that inhibition of HDAC1 and HDAC3 alleviates primarily the short-term motor skill learning deficits, accompanied by a partial repair effect on global gene expression changes in the striatum of R6/1 mice. MR imaging showed in addition, that RGFP109 treatment exerted modest changes on the atrophy of specific brain regions in the R6/1 mice. Collectively, these findings present evidence for beneficial effects of specific HDAC1 and HDAC3 inhibition on several aspects of disease pathology in HD mice. 
bioRxiv preprint doi: https://doi.org/10.1101/2020.10.13.337154; this version posted October 13,2020 . The copyright holder for this preprint (which was not certified by peer review) is the author/funder. All rights reserved. No reuse allowed without permission. 


\section{Results}

\section{HDAC1 and HDAC3 inhibition by RGFP109 ameliorates motor learning deficits typical of HD in $\mathrm{R} 6 / 1$ mice}

R6/1 transgenic mice faithfully recapitulate many of the disease features of human HD, such as transcriptional dysregulation, progressive impairments of both motor and cognitive functions (Brooks et al., 2012; Hodges et al., 2008; Naver et al., 2003; Yildirim et al., 2019), brain atrophy and mutant HTT accumulation (Bayram-Weston et al., 2012). In our study, to evaluate if the specific HDAC inhibitor RGFP109 could alleviate the transcriptional dysregulation in HD in vivo and improve the characteristic neuroanatomical HD features and behavioral deficits, we treated R6/1 mice (11 to 14 weeks of age) with $30 \mathrm{mg} / \mathrm{kg}$ (i.p.) RGFP109 five times a week for three weeks (total of 23 days) (Figure 1A). R6/1 mice display a range of characteristic behavioral deficits such as motor abnormalities, learning and memory impairments, and reduced level of anxiety (Brooks et al., 2012; Hodges et al., 2008; Naver et al., 2003). Thus, we examined the R6/1 mice using different behavioral tests including RotaRod, open field, and elevated plus maze during the course of the study (Figure $1 \mathrm{~A})$.

For evaluating the effect of HDAC1 and HDAC3 inhibition on motor coordination, balance, and motor skill learning in R6/1 mice, we performed the accelerating RotaRod test. Figure 1B (left) depicts the latency to fall as mean of four consecutive trials for each animal. Comparison of the vehicle-treated R6/1 and wild type animals revealed an HD-characteristic phenotype with significantly shorter time to stay on the rod in R6/1s. The latency to fall was significantly reduced by the main genotype effect $\left(\mathrm{F}_{1,40}=23.6\right.$ with $\left.\mathrm{p}<0.0001\right)$ while treatment had only a minor effect on motor coordination $\left(\mathrm{F}_{1,40}=1.09\right.$ with $\left.\mathrm{p}=0.304\right)$. However, mice with treatment stayed $26 \mathrm{~s}$ longer on the rod, albeit not being significant (mean of all trial: $101.8 \pm 44.2 \mathrm{~s}$ for R6/1-vehicle and $127.8 \pm 50.5 \mathrm{~s}$ for R6/1-RGFP109; p=0.651) (Figure 1B, left). This was also observed for the speed at fall (mean of all trials: 18.1 $\pm 5.7 \mathrm{rpm}$ for R6/1-vehicle and 21.4 $\pm 7.4 \mathrm{rpm}$ for R6/1-RGFP109; $\mathrm{p}=0.672$ ) (Figure S1A). In contrast, inhibition of HDAC1 and HDAC3 in R6/1 mice led to a significant improvement in RotaRod performance over the course of the four consecutive trials, indicating enhanced motor learning skills in HDACi-treated R6/1 animals ( $\mathrm{q}=0.017$ for trial 4 vs. trial 1 for latency to fall; $\mathrm{q}=0.026$ for trial 4 vs. trial 1 for speed at fall) (Figure 1B, middle and right). Notably, RGFP109 treatment in R6/1 animals resulted in a significant increase in time to stay on the rod compared to vehicle-treated R6/1s in the fourth trial $(103.1 \pm 40.0 \mathrm{~s}$ for R6/1-vehicle and $153.3 \pm 64.6 \mathrm{~s}$ for R6/1RGFP109 and a difference of $50.2 \mathrm{~s} ; \mathrm{q}=0.048$ ) (Figure 1B, middle). Albeit not statistically significant, a similar trend was observed for the speed at fall (mean difference=7.08; q=0.052) (Figure 1B, right). Vehicle-treated R6/1 mice as well as vehicle- and RGFP109-treated wild type animals did not exhibit 
significant changes in motor learning abilities during the four trials. These findings indicate that HDAC1 and HDAC3 inhibition by RGFP109 ameliorates motor learning deficits in R6/1 mice.

We next analyzed if the general locomotor activity in the open field was also affected by RGFP109 treatment in R6/1 mice. Locomotor activity is known to be impaired in the R6/1 line as well as in other HD models (Hodges et al., 2008; Naver et al., 2003). Accordingly, we observed a highly significant reduction in the total distance travelled as well as in the average velocity and vertical activity (supported rearing) in vehicle-treated R6/1 animals compared to vehicle-treated wild type littermates indicating a hypoactive phenotype in HD animals (Figure 1C, Figure S1B). However, no significant differences were found between vehicle- and RGFP109-treated R6/1 mice for any of these parameters. Next, we performed the elevated plus maze test, which has been developed for measuring anxiety in rodents (Walf and Frye, 2007). As previously described (Naver et al., 2003), R6/1 mice

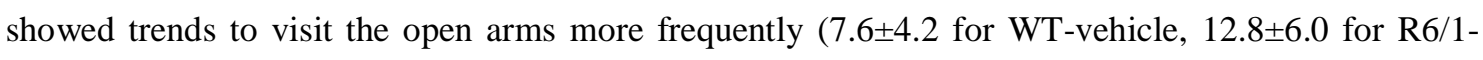
vehicle; $\mathrm{p}=0.137)$, spend more time $(13.6 \pm 11.2 \mathrm{sec}$ for WT-vehicle, $26.3 \pm 13.6 \mathrm{sec}$ for R6/1-vehicle; $\mathrm{p}=0.163)$, and travel longer distances $(38.8 \pm 29.2 \mathrm{~cm}$ for WT-vehicle, $78.0 \pm 45.5 \mathrm{~cm}$ for R6/1-vehicle; $\mathrm{p}=0.198$ ) in the open arms regardless of treatment. Taking the reduced locomotor activity of R6/1s into account, vehicle-treated R6/1 mice displayed a significantly less anxious phenotype than vehicletreated wild type mice (visits, time, and path in open arm normalized to total path). Treatment with RGFP109 did not exert a significant effect on this phenotype neither in R6/1 nor in wild type mice (Figure 1D, Figure S1C).

Lastly, abnormal limb clasping upon tail suspension can be used to demonstrate the presence of a neurological phenotype in HD mice. At the end of our study, before the sacrifice of mice (at 14-17 weeks of age), several R6/1 animals exhibited first degree forelimb clasping (score 0.5). However, RGFP109 administration did not affect the clasping behavior (Figure S1D). During the course of the study, body weight of the animals was not influenced by HDAC inhibitor administration indicating that the treatment was in general well tolerated. As expected, a reduction in the weight of the R6/1 mice was observed with disease progression, on which HDACi treatment showed no effect (Mangiarini et al., 1996; Naver et al., 2003) (Figure S1E).

Altogether, these results demonstrate that RGFP109 treatment alleviates primarily the motor skill learning deficits while not affecting the general locomotor activity or anxiety-related phenotypes in HD mice.

RGFP109 treatment alleviates, in part, the global gene expression changes in the striatum of R6/1 mice

Aberrant transcriptional regulation in brain, especially in striatum and cortex, is an early and central feature of HD pathogenesis. Among the genes with aberrant expression in HD striatum are transcriptional regulators (e.g. Fos, Egrl, Npas4, and Polr2a) as well as key neuronal genes important 
for neurotransmitter signaling (e.g. Drd1, Drd2, Grin3a, and Ppplrlb) and synaptic plasticity (e.g. Arc and Syp) (Seredenina and Luthi-Carter, 2012; Vashishtha et al., 2013; Yildirim et al., 2019). To examine the impact of RGFP109 treatment on transcriptional dysregulation, we conducted transcriptome analysis of the striatum by RNA-sequencing (RNA-seq) ( $\mathrm{n}=5$ for WT-vehicle, $\mathrm{n}=6$ for R6/1-vehicle, $n=6$ for WT-RGFP109, $n=7$ for R6/1-RGFP109). All animals were sacrificed $18 \mathrm{~h}$ after the final injection and the striatum was used for RNA isolation and subsequent analyses (Figure 1A).

Analysis of the RNA-seq data revealed that 1461 genes were significant differentially expressed in the R6/1 striatum at the age of sacrifice compared to wild type mice in the vehicle-treated group (FDR, $\mathrm{q}<0.1 ; \log 2 \mathrm{FC}>|0.5|$ ) with 1171 genes $(80 \%)$ being downregulated in R6/1 animals (Dataset S01). As expected, among these, key HD genes such as Drd1, Drd2, Ppp1r1b, Penk, and Adora2a were downregulated. Analysis of the RNA-seq data from the RGFP109-treated R6/1 striata showed that the HDACi treatment significantly changed the expression of 43 genes in R6/1 animals (FDR, $q<0.1$; $\log 2 \mathrm{FC}>|0.5|)$, of which 36 genes were also differentially expressed in R6/1s compared to wild types (Dataset S01). 14 out of these 36 genes were changed towards wild type expression levels upon RGFP109 treatment in R6/1 animals (Figure 2A, highlighted in red). Among these are transcription factor genes such as Neurod2, Neurod6, and Nr4a2, the transcriptional regulator Satb2, the thyrotropin-releasing hormone $T r h$, the neuropeptide-like molecule Nxph3, and the nicotinic acetylcholine receptor Chrna4 genes. We validated the RNA-seq findings by quantitative RT-PCR for several typical HD genes (Drd1, Drd2, Ppp1r1b, Adora2a, Arc, Egrl, Polr2a, and Grin3a), for the effect of HDAC1 and HDAC3 inhibition on the transcriptional changes in R6/1s (Nr4a2, Satb2, Folr1, and Otx2) (although not significant in qRT-PCR data), as well as for several genes that were previously connected to motor skill learning behavior in rodents (Dpysl2, Wars, and Cpne5) (D'Amours et al., 2011) in the striatum of vehicle- and RGFP109-treated wild type and R6/1 mice (Figure S2A).The complete lists of significant differentially expressed genes for the R6/1 and wild type comparison as well as for the treatment effect in R6/1 mice in RNA-seq data are presented in Dataset S01.

Further analysis of the datasets, without imposing cutoff criteria on the data for statistical significance, revealed a trend for a larger potential effect of RGFP109 treatment on expression of the dysregulated genes in R6/1 striatum, as illustrated in Figure 2A. Of the 1171 downregulated genes in the vehicle-treated R6/1s, expression of $21 \%$ (244) were enhanced by $>20 \%$ by the HDACi treatment of R6/1s and among these were genes such as adrenoceptor alpha 2a (Adra2a) and 1b (Adra1b), suppressor of cytokine signaling 3 (Socs3), and dual specificity phosphatase 6 (Dusp6), whose expression showed a shift towards wild type levels. Similarly, expression of 13\% (39) of the genes upregulated in the vehicle-treated R6/1 striatum were reduced by $>20 \%$ by the RGFP109 treatment and some of these genes were 5-hydroxytryptamine (serotonin) receptor $2 \mathrm{c}(\mathrm{Htr} 2 \mathrm{c})$, roundabout guidance receptor 1 (Robo1), and dopamine receptor d3 (Drd3) (Figure 2A and Figure S2B). To get a 
better functional view of genes that were alleviated by HDACi treatment in R6/1 animals, we performed gene ontology (GO) enrichment analysis of these genes that were changed towards wild type expression levels in RGFP109-treated R6/1 animals by at least 20\%. These genes were enriched for several GO terms that are relevant for brain physiological processes, such as neuropeptide hormone activity, regulation of secretion, and second-messenger-mediated signaling (Figure S2C). Dataset S02 shows the complete list of enriched GO terms (FDR, $\mathrm{q}<0.1$ ).

Next, we examined if expression levels of certain sets of genes associated with specific functions, phenotypes, or pathways would be more affected by HDAC1 and HDAC3 inhibition than individual genes. Using $\log 2$ fold changes for ranking genes, we performed gene set enrichment analysis (GSEA) to find the gene sets associated to the functional transcriptional changes in HD and those induced by HDAC1 and HDAC3 inhibition. Indeed, normalized enrichment scores of $43 \%$ (24 out of 55 ) of the gene sets that were negatively enriched in disease (R6/1-vehicle / WT-vehicle comparison) were changed by at least 5\% towards wild type levels in RGFP109-treated R6/1 mice (enrichment scores shifting towards 0 comparing drug-treated R6/1s vs. vehicle-treated wild type mice). Among these were gene sets associated with neuropeptide receptor binding, negative regulation of hormone secretion, and regulation of IFN $\alpha$ signaling, that were increased towards wild type levels. In contrast, among the positively enriched gene sets in R6/1, only protein dna complex and dna packaging complex were lowered towards wild type levels upon treatment (by $2.4 \%$ and $2.3 \%$, respectively). Of note, the neuroactive ligand receptor interaction gene set showed further enhancement of the diseaseassociated enrichment pattern upon HDAC1 and HDAC3 inhibition in R6/1 animals (by 6.4\%), while the remaining gene sets enriched in disease (31) were not changed or changed by less than $5 \%$ by RGFP109 treatment and these include actin filament based movement and potassium channel complex gene sets (Figure 2B, Dataset S02).

We next compared our results with a previous report which examined the effects of a similar HDACi, HDACi $4 b$, on genome-wide gene expression profiles in the striatum of R6/2 mice using microarray (Thomas et al., 2008). Of 56 genes, which were shown to be restored in the striatum by HDACi $4 \mathrm{~b}$ treatment of R6/2 mice, 42 were detected by RNA-seq in our study. Of these 42 genes, only 6 were significantly dysregulated in the striatum of R6/1 mice at the age of 14-17 weeks, which corresponds to a less progressed disease stage compared to the R6/2s, and 2 of these genes, Arhgap 15 and Gsto2, were significantly restored by RGFP109 treatment of the R6/1s in our study. To explore a more inclusive list of potentially therapeutically relevant genes, we examined all expression changes, regardless of their statistical significance, which revealed that of the 42 genes previously reported to be restored by HDACi 4b, 25 showed a change toward the WT levels after RGFP109 administration in R6/1 mice in our study (Figure 2C, Dataset S01), providing a list of genes whose expression levels are consistently ameliorated by specific HDAC1/3 inhibitors across different HD models. 
Collectively, RGFP109 treatment at least to a certain extent, repairs the transcriptional effects of mutant Huntingtin gene expression, causing a significant change in the expression of 43 genes, in the striatum of R6/1 mice and induced collective changes in the expression of gene sets, some of which were associated to biological functions and processes that are relevant for neuronal physiology and HD pathogenesis.

\section{Effects of RGFP109 treatment on the HD-associated atrophy of specific brain areas in the R6/1 mice measured by volumetric MRI}

One hallmark of HD is neuronal degeneration which manifests predominantly in the striatum, particularly the caudoputamen and dorsal striatum in HD patients. In addition, brain atrophy of variable severity can be observed in several other brain regions, such as the cerebral cortex, total white matter, amygdala, hippocampus, and brainstem (Rosas et al., 2003; Tabrizi et al., 2012). The R6/1 mouse model closely recapitulates the neuronal degeneration seen in HD patient brain. These mice exhibit progressive brain atrophy in the striatum (especially the posterior striatum) and cortex (especially in the retrosplenial areas) as well as a subtle expansion of posterior ventricular spaces (Rattray et al., 2013).

By registering the Allen brain atlas to T2w MR images (Koch et al., 2019) we used an unbiased approach to detect regional and sub-regional changes in R6/1 mice upon RGFP109 treatment (Figure 1A). This analysis revealed 419 brain regions and sub-regions (55\% of all analyzed regions) that showed a significant volume difference between vehicle-treated R6/1 and wild type mice at the age of 13-16 weeks of age (FDR, q<0.1) (Figure 3A, Figure S3A). Of these, the majority (403 regions, 96\%) exhibited a significant volume decrease in R6/1 mice including striatum, pallidum, hippocampal region, and piriform area. In contrast, 16 regions (4\%) showed a significant volume increase in HD mice, e.g. copula pyramidis, orbital area (medial part, layer 1 and 2), and supratrigeminal nucleus (Figure S3E and Dataset S03). RGFP109 treatment did not induce any significant changes in the volumes of the afore-mentioned 419 brain substructures in R6/1 mice at this age. However, 61 regions (15\%) with significant decrease or increase in R6/1 animals showed non-significant trends towards a reversal of the volume change upon drug treatment by at least 20\% (Figure 3A, framed red). Some of these regions include central amygdalar nucleus (medial part), pallidum (ventral region), and prelimbic area. Dataset S03 contains the complete list of brain regions with significant volume difference in R6/1 animals and those that are impacted by the treatment.

As the overall brain volumes in R6/1 mice were reduced (Figure S3E, first graph), we utilized a second approach that corrects the regional and sub-regional volumes by the whole brain volume. This resulted in 220 regions (29\% of all analyzed regions) showing a significant change in R6/1 animals (Figure 3B, Figure S3B). 144 brain regions with significant volume change were shared between the two analysis strategies (Figure S3C). Similar to the absolute volume analysis, RGF109 treatment did 
not show any significant effects on volumes of brain regions in R6/1 mice after correcting for the whole brain atrophy. Non-significant trends towards an improved volume change of at least $20 \%$ upon drug treatment in R6/1 mice was detectable for 25\% (55/220) of the regions showing a significant volume difference in R6/1 mice (Figure 3B, framed red). 17 brain regions and sub-regions positively affected by drug treatment by more than $20 \%$ in R6/1s were shared in both analysis strategies. These include piriform area (molecular layer), ectorhinal area, intermediodorsal nucleus of the thalamus, and prelimbic area (layer 5) (Figure S3D, Figure S3E, Dataset S03). Figure 3C depicts selected regions that were previously implicated in motor skill learning (amygdala, caudoputamen, ectorhinal cortex, entorhinal cortex, pallidum, piriform area, primary motor cortex) in representative examples of wild type, R6/1 and HDACi-treated R6/1 mice (Badea et al., 2019; Scholz et al., 2015; Tamakoshi et al., 2014).

In summary, matching the Allen brain atlas to MR images allowed us to investigate the HD mouse neuroanatomy and the impact of RGFP109 treatment on it in great detail. While we detected a large number of brain regions and sub-regions with significant volume change in R6/1 animals, HDAC1 and HDAC3 inhibition exhibited only trends towards slowing these volumetric differences in the brains of $\mathrm{R} 6 / 1$ mice at this progressed disease stage.

\section{RGFP109 treatment does not affect aggregate formation or bulk histone H3K27acetylation levels in R6/1 mice}

HD is characterized by mutant HTT protein aggregate formation, which displays a histopathological basis of transcriptional dysregulation, neuronal degeneration, and behavioral deficits. Given the effect of RGFP109 on short-term motor skill learning, we examined whether RGFP109 treatment has an impact on this neuropathological hallmark of HD. For this purpose, we analyzed EM48 immunoreactivity in striatal sections visualizing aggregated mutant HTT. We analyzed 8 separate images per section, each covering a $34192 \mu \mathrm{m}^{2}$ area with 123 DAPI+ cells in average in the dorsal stratum directly lateral to the lateral ventricle. By using ImageJ software, we defined particles larger than $15 \mu \mathrm{m}^{2}$ as nuclei. Blinded manual counting of the number of aggregates showed that RGFP109 did not affect the number of mutant HTT protein aggregates in R6/1 mice (Figure S4A).

To examine the effects of RGFP109 treatment on global histone acetylation, we determined bulk acetylation patterns at lysine 27 of histone $\mathrm{H} 3(\mathrm{H} 3 \mathrm{~K} 27 \mathrm{ac})$ in protein extracts of striatal tissue via immunoblotting. Increased H3K27ac levels can be found at active promoters and enhancers indicating high transcriptional activity (Wang et al., 2008). Our Western blotting analysis showed no differences in acetylated H3K27 levels between R6/1 and wild type mice, regardless of treatment. As control for equal histone amounts, we determined total H3 levels from the same samples. Similarly, we did not observe differences between the groups (Figure S4B). While we did not detect increases in global $\mathrm{H} 3 \mathrm{~K} 27 \mathrm{ac}$ patterns in striatum by the HDACi treatment, temporally dynamic and site-specific changes 
in acetylation levels not detected by immunoblotting of total tissue could be associated to the transcriptional changes observed in HD.

\section{Discussion}

This study shows that treating HD mice with a specific HDAC1 and HDAC3 inhibitor has beneficial effects on multiple aspects of HD. Inhibiting histone modifying enzymes, in particular class I HDACs, has been previously shown to improve disease features, such as the phenotypic and neuropathological deficits and dysregulation of selected genes, in various models of HD (Chen et al., 2013; Chiu et al., 2011; Jia et al., 2012; Jia et al., 2016; Lim et al., 2011; Suelves et al., 2017; Thomas et al., 2008). The goal of our study was to evaluate the effects of the specific HDAC1 and 3 inhibitor RGFP109 in an unbiased approach on the genome-wide transcriptional dysregulation and on the phenotypic deficits in HD mice using the R6/1 model. Further, by performing MRI measurements on living mice, we conducted a detailed analysis of the brain volumetric changes in the R6/1 mice. Our findings demonstrate that the specific inhibition of HDAC1 and HDAC3 by RGFP109 improves primarily the motor learning skills and alleviates, at least to a certain extent, the dysregulation of a number of genes and gene sets in the R6/1 mice. By volumetric MRI, we detected extensive atrophic changes in the brains of the R6/1 mice, on which RGFP109 exerted a statistically non-significant modest effect. Given our results and data from others, early targeting of transcriptional dysregulation by specific HDAC inhibition may alleviate key aspects of HD pathology.

In a previous study, a short-term treatment regime with RGFP109 (subcutaneous injections of $150 \mathrm{mg} / \mathrm{kg} /$ day for three days) partially reversed four selected disease-associated genes in the striatum in HD R6/2 mice as measured by PCR (Jia et al., 2012). However, the effects of RGFP109 treatment on the behavioral deficits and on gene expression throughout the genome as well as on the brain atrophy have not been evaluated in HD. Here, using the R6/1 mice, which is less progressive than the R6/2 model, we chose to use a lower therapeutic dose of $30 \mathrm{mg} / \mathrm{kg}$ based on a previous Parkinson's disease study (Johnston et al., 2013) and applied a longer treatment regime (three weeks with five injections per week), aiming to minimize potential toxic side effects by reducing high drug exposure.

Among the most characteristic behavioral deficits in HD mice are motor impairment and deficit in motor skill learning, which are caused by the dysfunction of corticostriatal circuits (Backman et al., 1997; Cybulska-Klosowicz et al., 2004; Lawrence et al., 2000; Mazarakis et al., 2005). The RotaRod task is a useful marker for detecting early HD phenotypes in R6/1 animals from as early as 8 weeks of age (Brooks et al., 2012). Our study demonstrates that 3-week-long treatment with $30 \mathrm{mg} / \mathrm{kg}$ RGFP109 ameliorates motor skill learning deficits in this mouse model of HD. While we obtained 
this finding by testing the animals on the RotaRod for up to four trials on the same day, in future studies, it would be important to analyze the animals across several days to assess learning over longer time frames as well as to use modified RotaRod protocols or other behavioral tests to better distinguish motor skill learning from motor coordination and balance. In line with our findings, Suelves et al. demonstrated that selective HDAC3 inhibition by RGFP966 in the $\mathrm{Hdh}^{\mathrm{Q} 7 / \mathrm{Q} 111}$ knock-in mouse model of HD (50 mg/kg of RGFP966 three times per week from 3 to 6.5 months) prevents corticostriatal-dependent motor learning deficits from trial four on testing animals for four times per day for three consecutive days (Suelves et al., 2017). Similarly, treatment of N171-82Q HD mice with RGFP966 over a period of 10 weeks with 10 or $25 \mathrm{mg} / \mathrm{kg}$ per week showed improved motor function, accompanied by neuroprotective effects on striatal volume, and significant alterations of the expression of 3 immune pathway genes (chemokine (C-C motif) ligand 17 (Ccl17), macrophage migration inhibitory factor (Mif), interleukin 13 (Il13); measured by PCR) (Jia et al., 2016). Notably, in the latter study, the beneficial effects of RGFP966 on the RotaRod task was observed in female HD mice only, suggesting potential differences in HDACi's effects depending on sex. While a sex comparison was not within the scope of our study, it would be important to test RGFP109, which showed therapeutic effects in male R6/1 mice in this current study, also on female R6/1s in future. In a mouse model of Friedreich ataxia prolonged RGFP109 treatment led to modest improvements in motor coordination performance and locomotor activity (Sandi et al., 2011). It has been shown that improvement on the RotaRod mainly requires a change in the motor strategy to master the task rather than an enhancement in general locomotor activity (Buitrago et al., 2004). Accordingly, we did not observe improvement in general locomotor activity in the open field test upon RGFP109 treatment in R6/1 animals.

To investigate the potential molecular correlates of our behavioral findings, we carried out a genomewide analysis of the transcriptional changes, which was not done before in the afore-mentioned studies, in the striata of vehicle- and drug-treated R6/1 and wild type mice. Our RNA-seq data showed the altered expression of a large number of genes in the striatum of R6/1 mouse, which included also those that are well-known to be dysregulated in HD, e.g. Drd1, Drd2, Penk and Adora2a (Seredenina and Luthi-Carter, 2012). RGFP109 administration showed, in part, repair effects on the global aberrant gene expression changes in the striatum of R6/1 mice, affecting 43 individual genes significantly. Some of these genes were the thyrotropin-releasing hormone gene Trh, the neuropeptide-like molecule gene $N x p h 3$, the nicotinic acetylcholine receptor gene Chrna4, and transcriptional regulator genes such as Neurod2, Neurod6, Nr4a2 and Satb2, whose change in expression may likely result in alterations in the expression of their target genes. Further, Arhgap15 (Rho GTPase activating protein 15) and Gsto2 (Glutathione S-Transferase Omega 2), genes whose expression were completely rescued in a previous report by the HDAC1 and HDAC3 selective inhibitor HDACi 4b in R6/2s (Thomas et al., 2008), were also restored in our study upon RGFP109 
treatment in R6/1s, supporting a similar mode of action of these selective HDAC inhibitors across different models of HD. Of note, loss of Arhgap15 gene, a member of the Rac signaling pathway, was shown to cause decreased synaptic density and cognitive deficits in mouse and its mutations were identified in association with neurological and cognitive deficits in patients with Intellectual Disability (Zamboni et al., 2018), revealing the rescue of its expression by HDACi treatment as potentially therapeutically relevant in HD. Beyond these single gene expression changes, we found that a number of gene sets associated with neurophysiological functions relevant for HD pathology were changed upon treatment and these changes could be linked to the observed phenotypic changes. Also, it should be taken into consideration that gene expression changes restricted to certain cell types in the striatum may have been diluted in RNA-seq measurements that use total striatal tissue preparations as in the present study.

In an animal study of Friedreich Ataxia it was shown that long-term RGFP109 treatment $(100 \mathrm{mg} / \mathrm{kg}$ over a period of five months with five injections per week) increased especially local H3K9ac and $\mathrm{H} 4 \mathrm{~K} 5 \mathrm{ac}$ levels directly at the frataxin gene accompanied by higher frataxin gene expression levels in mouse brain, whereas global $\mathrm{H} 3$ and $\mathrm{H} 4$ acetylation patterns did not significantly increase by the drug (Sandi et al., 2011). Next to the spatial changes of specific histone acetylation patterns, temporal differences of global and local histone acetylation have been observed in another Friedreich Ataxia mouse study using a single RGFP109 administration (with $150 \mathrm{mg} / \mathrm{kg}$ ) (Rai et al., 2010). In this study, global $\mathrm{H} 3$ acetylation in the brain increased to a maximum level at 4 hours after injection and totally disappeared at 24 hours, whereas $\mathrm{H} 4 \mathrm{~K} 5 \mathrm{ac}$ and $\mathrm{H} 3 \mathrm{~K} 14 \mathrm{ac}$ at the frataxin gene were shown to increase between 12 and 24 hours (Rai et al., 2010). In contrast to the aforementioned studies in mouse models of Friedreich Ataxia, we did not observe increased global H3K27 acetylation by RGFP109 treatment measured by Western blotting of total striatal extracts. Supporting our findings of no change in global histone acetylation after RGFP109 treatment, a previous report showed histone acetylation changes only at specific promoters using a similar HDAC inhibitor, HDACi $4 \mathrm{~b}$, in the R6/2 mice (Thomas et al., 2008).

Aiming at examining different key features of HD, on which RGFP109 treatment may have an effect, we performed MRI measurements to study the structural brain changes in HD mice. By registering the Allen brain atlas to MR images, we provided a complete list of regional and sub-regional volumetric changes of the brains of R6/1 animals, which by far expands the set of regions so far shown to be changed in this HD model. Using longitudinal in vivo MRI, a previous study detected reduction in both global brain volume as well as brain sub-regional volumes when corrected for global volume change in R6/1 mice over time, showing ubiquitous shrinkage of the striatum and the somatosensory cortices (Rattray et al., 2013), comparable with what we detected with our approach here. In contrast, increases in regional and sub-regional volumes when corrected for whole brain volume should be viewed cautiously, as they indicate a less pronounced shrinkage of that brain area compared to the 
volume reduction of the whole brain, rather than an actual regional size increase. In pre-symptomatic and symptomatic HD patients progressive whole brain volume loss is evident (Tabrizi et al., 2012). Furthermore, in addition to the striatum, other brain regions known to be affected in HD patients, such as cerebral cortex, amygdala, hippocampus, and brainstem (Rosas et al., 2003), are also changed in R6/1 mice. Similar to the modest transcriptomic changes we observed upon RGFP109 treatment, volumes of specific brain regions and sub-regions showed only non-significant trends towards an alleviation upon HDAC1 and HDAC3 inhibition in R6/1 mice at this disease stage. Of note, these regions include some of the brain areas that were previously implicated in motor skill learning behavior in rodents, such as the amygdala, ectorhinal cortex, entorhinal cortex, pallidum, piriform area, and primary motor cortex (Badea et al., 2019; Scholz et al., 2015; Tamakoshi et al., 2014).

In summary, our study demonstrates that treatment with the HDAC inhibitor RGFP109 provides benefits on key phenotypic and molecular aspects of HD as well as potential effects on the brain atrophy observed in the R6/1 mice. Further studies should include also relevant immunohistochemical assessments of the brain for elucidating potential synaptic plasticity changes that may contribute to the observed phenotypic effects of HDAC inhibition in HD mice. Although the R6/1 line recapitulates several key features of $\mathrm{HD}$, such as motor and cognitive deficits, transcriptional dysregulation, accumulation of mutant $\mathrm{Htt}$ aggregates and brain atrophy, establishing direct mechanistic links between molecular pathology and specific behavioral deficits has been a challenge so far (Rattray et al., 2013). Further work using an HD mouse model with slower progression, intervention earlier in the time course of pathology, a longer treatment regime with various doses or a combination of these factors may enhance the leveraging of the therapeutic potentials of selective HDAC inhibitors in HD. Nevertheless, we have demonstrated that HDAC1/3 inhibitor RGFP109 improved motor skill learning deficit and alleviated transcriptional dysregulation, characteristic disease features, in HD mice. 


\section{Author contributions}

K. H. and F.Y. designed research, K. H., S. M. and M. H. M. performed research, K. H., S. M., S. P. K., C.H., B.K. and P. B.-S. analyzed data, and K. H. and F. Y. wrote the manuscript.

The authors declare no conflict of interest.

\section{Acknowledgements}

This work was funded by Deutsche Forschungsgemeinschaft (DFG), Exc 257 NeuroCure (to F.Y.). Funding to S.M., S.P.K., CH and P.B.S. was provided by the German Federal Ministry of Education and Research (BMBF, Center for Stroke Research Berlin 01EO1301), the BMBF under the ERA-NET NEURON scheme (01EW1811) and the DFG (research grant BO 4484/2-1 and HA5741/5-1 to CH). We thank the Department of Experimental Neurology, Charité - Universitätsmedizin Berlin for support with behavioral analyses, the Core Facility 7T Experimental MRIs, Charité Universitätsmedizin Berlin for support with MRI measurements, the Scientific Genomics Platforms, Max-Delbrück-Center for Molecular Medicine (MDC) in the Helmholtz Association, Berlin for sequencing, SPARK Berlin for scientific advice, Heike Lerch and Cansin Belgin Peksen for excellent technical assistance, and Theresa Hartung for critically reading the manuscript. 


\section{Materials and Methods}

\section{Animals}

Hemizygous R6/1 mice, expressing exon 1 of the human HTT gene with 116 CAG repeat expansions, were purchased from the Jackson Laboratory. R6/1 mice were maintained on a C57BL/6J background crossing male R6/1 with female C57BL/6J. Genotypes were determined by PCR analyses (R6/1-fwd: 5'-CCGCTCAGGTTCTGCTTTTA-3'; R6/1-rev: 5'-GGCTGAGGAAGCTGAGGAG-3'). Littermates were randomly divided into four groups: WT-vehicle $(n=10)$, R6/1-vehicle ( $n=13)$, WTRGFP109 (n=10), R6/1-RGF109 ( $n=12)$. All mice used in the present study were housed together in groups of maximal four animals; if possible, genotype and treatment were mixed in individual cages. Weight of the mice were monitored throughout the study. No animal was excluded due to excess weight loss (exclusion criterium: $\geq 25 \%$ weight loss compared to the weight at the start of the experiment). One animal (R6/1-vehicle) died during the course of the experiment (after completion of the MRI measurement and open field test, before performing Rotarod and elevated plus maze tests) due to unknown reasons. Animals were housed under pathogen free conditions with ad libitum access to food and water on a $12 \mathrm{~h}$ light/12 $\mathrm{h}$ dark cycle at constant temperature $\left(22 \pm 2{ }^{\circ} \mathrm{C}\right)$ and humidity $(55 \pm 10 \%)$. All animal experiments were approved by the local animal care committee of CharitéUniversitätsmedizin Berlin and by the Landesamt für Gesundheit und Soziales Berlin (license number G0314/16) and conducted according to the institutional guidelines. All efforts were made to minimize all unnecessary suffering of animals. In line with our license for animal experiments and the $3 \mathrm{Rs}$ principles, for reducing the data variation and thereby keeping the required number of mice for the experiments to a minimum, only male mice were used in this study.

\section{RGFP109 treatment of mice}

The HDAC1 and HDAC3 inhibitor RGFP109 (RG2833; CAS No. 1215493-56-3) was purchased from Selleckchem. RGFP109 was dissolved in dimethyl sulfoxide and diluted in $0.9 \% \mathrm{NaCl}(1: 2)$ directly before use (final concentration: $15 \mathrm{mg} / \mathrm{ml}$ ). Mice were administered RGFP109 (30 mg/kg body weight) or an equal volume of vehicle solution by intraperitoneal injections five times a week for three weeks (total of 23 days) starting at the age of 11 to 14 weeks. All injections and behavioral tests were done around the same time of the day to avoid any biochemical and physiological changes over the experiments. Mice were deeply anesthetized using isoflurane and sacrificed by cervical dislocation $18 \mathrm{~h}$ after the final injection. Brains were removed and separated into right and left hemispheres. One hemisphere was flash frozen at $-80{ }^{\circ} \mathrm{C}$ in methyl butane (Sigma-Aldrich) for immunohistochemical analysis, one was further dissected for RNA extraction and immunoblot analyses of striatum. 


\section{Behavioral assessment}

Motor coordination, balance as well as motor skill learning were assessed by accelerating RotaRod performance test. The open field test was used to examine general locomotor activity. Mouse emotional state, fearfulness, arousal, and anxiety were evaluated by the elevated plus maze test assessing exploration and motor activity in a new open environment. On the day of the individual tests, animals were moved to the test room 30 minutes prior to the start of the test to allow sufficient time to habituate. All data recorded, regardless of individual behavior, were used for the analyses.

At the end of the treatment course, mice were tested for forelimb and hindlimb clasping behavior by suspending each mouse by the tail, $20 \mathrm{~cm}$ above their home cage for up to 30 seconds (lack of any clasping behavior scored 0 ; rapid movement of forelimbs scored 0.5 ; forelimb clasping behavior towards the abdomen scored 1 ; forelimb and hindlimb clasping behavior towards the abdomen scored 2).

Accelerating RotaRod One day before the actual test, mice were trained on the RotaRod (TSE Systems) three times at constant speed ( $4 \mathrm{rpm}$ for 60 seconds) and two times with accelerating speed ( 4 to $40 \mathrm{rpm}$ over a period of 5 minutes) with 30 minutes interval between the sessions. During the training mice were placed back on the rod if they have fallen down. On the following day data were recorded for four test trials using the TSE RotaRod software, which detects the latency to fall in seconds and the rod rotational speed at fall in rpm. The test trials were done by accelerating the rod from 4 to $40 \mathrm{rpm}$ over a period of 5 minutes with at least 30 minutes interval between the sessions. The rod was cleaned between animal trials to remove any odors.

Open Field The open field test was performed in a grey open-top $50 \times 50 \mathrm{~cm}$ arena (height $40 \mathrm{~cm}$ ) located in a sound-attenuated observation chamber. Mice were individually placed near the wall of the box and locomotor activity was measured as total distance travelled and average velocity over a period of 10 minutes. VideoMot2 Software, TSE Systems was used to track and record all animals (standard measuring mode based on center of gravity). Vertical activity (supported rearing) was manually assessed analyzing the recorded videos (experimenter was blinded for treatment and genotype). The field was thoroughly cleaned between the animals to avoid any odor.

Elevated plus maze The elevated plus maze test was performed in a sound-attenuated observation chamber. The maze configuration consisted of five main regions: two open arms $(29.5 \times 5 \mathrm{~cm})$, two closed arms $(29.5 \times 5 \mathrm{~cm})$ and a middle area connecting the arms. The plus maze was raised $68 \mathrm{~cm}$ above the floor. Animals were placed in the center of the maze towards one of the closed arms. 
Movements were tracked (standard measuring mode based on center of gravity) and recorded for 5 minutes using VideoMot2 Software (TSE Systems). Number, duration, and path of visits to the open arms were calculated. The maze was rigorously cleaned between the animals to remove any scent clues left by the previous subject mouse.

\section{MRI measurements}

MRI measurements were performed under 1-2\% isoflurane anesthesia in a 70:30 nitrous oxide:oxygen mixture. Temperature was maintained through a circulating warm water system. Respiration rate was monitored during the measurements (Small Animal Instruments, Inc., Stony Brook, NY). T2weighted images were acquired on a 7T MR scanner (PharmaScan 70/16 US; Bruker, Ettlingen, Germany) using a $20 \mathrm{~mm}$ quadrature volume resonator (Rapid Biomed). To cover the whole brain, a 2D T2-weighted RARE pulse sequence was used with 32 contiguous axial slices with $0.5 \mathrm{~mm}$ slice thickness and in-plane field of view of 25.6 x $25.6 \mathrm{~mm}$. The imaging parameters were: matrix size 256x256, echo time spacing $\Delta \mathrm{TE}=12 \mathrm{~ms}$, repetition time $\mathrm{TR} /$ effective echo time $\mathrm{TE}=4200 / 36 \mathrm{~ms}$, band width $=46875 \mathrm{~Hz}$, RARE factor 8, 4 averages, acquisition time 6:43 $\mathrm{min}$.

The Allen brain atlas (Lein et al., 2007) was registered to individual MR images using the MATLAB toolbox ANTX (Koch et al., 2017) and the volume of each brain region was measured in $\mathrm{mm}^{3}$. For statistical comparison of brain region volume between groups a t-test (FDR, $q<0.1$ ) was applied. Only regions with a size of $>0.1 \mathrm{~mm}^{3}$ (corresponding to 20 voxels) in at least one of the analyzed groups were included in the analysis. Both, absolute brain region volumes as well as brain region volumes normalized to whole brain volume were evaluated.

\section{Gene expression analysis by RNA-seq, RNA-seq data analysis, and qRT-PCR}

RNA isolation Flash-frozen tissues were homogenized in QIAzol Lysis Reagent (Qiagen) followed by RNA extraction using the miRNeasy Kit (Qiagen). Libraries for RNA-seq analysis were prepared using the TruSeq RNA kit from Illumina. Two pools with twelve libraries each (pool 1: WT-vehicle $(n=3)$, R6/1-vehicle ( $n=3)$, WT-RGFP109 ( $n=3)$, R6/1-RGF109 ( $n=3)$; pool 2: WT-vehicle $(n=2)$, R6/1-vehicle ( $n=3)$, WT-RGFP109 ( $n=3)$, R6/1-RGF109 $(n=4)$ ) were sequenced on a Illumina HiSeq4000 instrument (1x51bp).

RNA-seq mapping and analysis Raw single-end reads of cDNA fragments were aligned to the mouse transcriptome (RefSeq, mm10) using the 'RNA-Seq Alignment Workflow' from BaseSpace, Illumina (version 1.1.0) with STAR aligner (version STAR_2.5.0b) for mapping and Cufflinks (version 2.2.1) for fragments per kilobase of exon per million fragments mapped (FPKM) estimation of reference genes. Differential gene expression analysis was performed using 'Cufflinks Assembly \& DE 
Workflow from BaseSpace, Illumina (version 2.1.0) with Cuffdiff 2 (Cufflinks, version 2.2.1). Differential expressed genes with FDR, $\mathrm{q}<0.1$ were considered significant.

Gorilla analysis Functional enrichment for gene ontology terms (biological process, molecular function, and cellular component) were calculated using the two unranked list approach (target and background lists) from Gorilla (Eden et al., 2009) . As target gene list all genes significantly different in R6/1-vehicle / WT-vehicle comparison (FDR, $\mathrm{q}<0.1$; $\log 2 \mathrm{FC}>|0.5|$ ) and affected by RGFP109treatment in R6/1 mice by at least 20\% (283) were used. All expressed genes, with FPKM $>0.1$ in at least one sample (18844) were used as background.

Gene set enrichment analysis For gene enrichment analysis only genes with FPKM>0.1 in at least one of the groups analyzed were used. Pseudocounts of 0.05 FPKM were added to every gene to circumvent inflated fold changes at low expressed genes. Mouse gene symbols were converted into human gene symbols using gene IDs from BioMART-Ensemble (https://www.ensembl.org/biomart). Enriched genes sets were analyzed using the software from Broad Institute (GSEA, (Mootha et al., 2003; Subramanian et al., 2005) with MgSigDB gene set collections for gene ontology, KEGG, and REACTOME pathways). 1000 gene-set-wise permutations were performed to generate the null distributions. Genes were ranked in descending order based on $\log 2$ fold changes. Enrichment scores were calculated using the classic statistic (unweighted). Gene sets with FDR, $\mathrm{q}<0.1$ were considered significant.

Quantitative PCR cDNA synthesis was performed with M-MLV reverse transcriptase (Promega) and random hexanucleotides. mRNA expression levels were assessed by quantitative real-time PCR using SYBR Green dye-based PCR amplification (Thermo Fisher Scientific) and the QuantStudio 3 detection system (Applied biosystems). Primer sequences are listed in Table S1. mRNA expression levels were calculated relative to housekeeping gene Actb according to following equation: $2^{\text {(Ct(Actb)- }}$ $\mathrm{Ct}($ target gene))

\section{Immunoblot analysis}

Flash-frozen tissues were homogenized in RIPA buffer (50 mM Tris pH7.4, $150 \mathrm{mM} \mathrm{NaCl}, 1 \%$ Triton $\mathrm{X}-100,0.1 \%$ SDS, $1 \%$ sodium deoxycholate and protease inhibitor cocktail (Thermo Scientific)), incubated for $2 \mathrm{~h}$ at $4{ }^{\circ} \mathrm{C}$, sonicated and clarified (12,000 x g). Equal amounts of protein $(20 \mu \mathrm{g})$ were subjected to SDS-PAGE and subsequently transferred to a nitrocellulose membrane. Membranes were blocked and then incubated with primary antibodies (anti-H3K27ac, abcam; anti-H3, Cell Signaling Technology; anti- $\beta$ Actin, Cell Signaling Technology) at $4^{\circ} \mathrm{C}$ overnight. Staining with secondary HRP-conjugated antibody was performed at room temperature for $1 \mathrm{~h}$. Detection of the membrane was carried out using SuperSignal ${ }^{\mathrm{TM}}$ West Dura Extended Duration Substrate (Thermo Fisher Scientific). Histone intensities were compared and normalized to beta-Actin intensities from the same blot using ImageJ (ImageJ software, NIH). 


\section{Immunohistochemistry}

The hemispheres were cut in coronal sections of $30 \mu \mathrm{m}$ using a Leica CM1950 cryostat. Sections were dried on glass slides at room temperature before fixating in an Aceton:Methanol (1:1) solution for $10 \mathrm{~min}$ at $-20^{\circ} \mathrm{C}$. After a short drying period, sections were rehydrated in PBS and blocked for $1 \mathrm{~h}$ in $10 \%$ goat-serum in PBS with $0.1 \%$ Triton-X 100. Huntington aggregates were stained at $4{ }^{\circ} \mathrm{C}$ over night (EM48, Millipore; 1:500, diluted in 10\% goat-serum in PBS). Slides were washed in PBS and incubated with a secondary antibody (anti-mouse, Rhodamine Red-X- conjugated, Jackson ImmunoResearch; 1:1000, diluted in 10\% goat-serum in PBS). After washing sections were stained with DAPI, washed, and mounted in Immumount.

Image acquisition was performed using a Nikon Spinning Disk Confocal CSU-X microscope equipped with a Nikon Plan Fluor 40x/1.3 DIC H N2 objective and Andor iXon3 EMCCD DU-888 Ultra camera. Images were acquired using Nikon NIS-Elements (version 5.10) imaging software.

For HTT-aggregate quantification, 8 spatial separated z-stack images (21 images with $500 \mathrm{~nm}$ z-steps) of one striatal section per animal were acquired and analysed. HTT aggregates were counted manually based on maximum intensity projections of each stack (ImageJ software, NIH). Experimenter was blinded for treatment and genotype. Individual nuclei within tissue sections were identified using the DAPI signal. Using ImageJ, sum slice projections were created for each z-stack image followed by background signal subtraction (rolling ball radius: 15 pixels). A binary image was created, and the watershed method was used to separate touching objects. Particles larger than $15 \mu \mathrm{m}^{2}$ were defined as nuclei.

\section{Statistical Analysis}

Behavioral, qPCR, immunohistochemistry, and immunoblot data were analyzed using the Graphpad Prism 6.0 and 8.0 software. The statistical tests included unpaired, two-tailed Student's $t$-test, , twoway ANOVA followed by Tukey's multiple comparisons test (for measuring the response to genotype and treatment), and mixed-effects analysis with two-stage linear step-up procedure of Benjamini, Krieger and Yekutieli as post-tests (for repeated measures data) as indicated in each figure legend. Statistical comparisons, values for $\mathrm{n}$ and $\mathrm{p}$ (unpaired, two-tailed Student's $t$-test and two-way ANOVA) or q (mixed-effects analysis) are indicated in the figure legends. Differences with $\mathrm{p} \leq 0.05$ or $\mathrm{q} \leq 0.05$ were considered significant. Visualizations of RNA-seq and MRI data and their statistical results were done in $\mathrm{R}$ or with Graphpad Prism 6 software. We use the term 'trend' for referring to non-significant changes that are below the significance threshold in the respective experiments. 
bioRxiv preprint doi: https://doi.org/10.1101/2020.10.13.337154; this version posted October 13,2020 . The copyright holder for this preprint (which was not certified by peer review) is the author/funder. All rights reserved. No reuse allowed without permission. 


\section{Figure Legends}

Figure 1. RGFP109 treatment improves motor learning in R6/1 mice. (A) Schematic of treatment and experimental procedure. (B) Motor performance and learning on a RotaRod: Latency to fall as mean of all trials, latency to fall and speed at fall as learning curve over the course of four subsequent trials. (C) Open field exploration evaluating general locomotor activity measured as total distance travelled. (D) Anxiety-related behavior determined by elevated plus maze test showing visits to open arm and path travelled in open arm normalized to the total path. WT - wild type. Data represent mean+SD; n=10 [WT-vehicle], n=12(13, Open Field) [R6/1-vehicle], n=10 [WT-RGFP109], n=12 [R6/1-RGF109]; *p $\leq 0.05, * * \mathrm{p} \leq 0.01, * * * \mathrm{p} \leq 0.001, * * * \mathrm{p} \leq 0.0001$, ns not significant by two-way ANOVA with Tukey's multiple comparisons test (B-left graph, C, and D); ${ }^{*} \mathrm{q} \leq 0.05,{ }^{\#} \mathrm{q} \leq 0.01$, ns not significant by mixed-effects analysis with two-stage linear step-up procedure of Benjamini, Krieger and Yekutieli as post-tests by controlling the False Discovery Rate (individually comparing each group to each other group) (B-learning curves: comparison of trial 4 between groups - indicated vertically, comparison of trial 4 vs. trial 1 for R6/1-RGFP109 - indicated horizontally).

Figure 2. Inhibition of HDAC1 and HDAC3 by RGFP109 has a partial repair effect on global gene expression changes in the striatum of R6/1 mice. (A) 283 genes, significant differentially expressed between WT and R6/1 mice (black) (Cuffdiff 2; FDR, q<0.1; $\log 2 \mathrm{FC}>|0.5|$ ), are changed towards wild type expression levels in RGFP109-treated R6/1 animals by at least $20 \%$ using no cutoff criteria on the data for statistical significance (green). Significant differentially expressed genes upon RGFP109 treatment in R6/1 mice (R6/1-RGFP109 / R6/1-vehicle; FDR, $\mathrm{q}<0.1$; $\log 2$ FC $>|0.5|$ ) are framed in red. Selected genes are labeled. (B) Gene set enrichment analysis (GSEA - Broad Institute) for gene ontology, KEGG pathway, and REACTOME pathway gene sets. Genes were ranked based on $\log 2$ fold changes. The bar graph shows gene sets with significant different normalized enrichment scores (NES) between WT-vehicle and R6/1-vehicle mice (FDR, q<0.1). NESs of RGFP109-treated R6/1 compared to WT-vehicle mice are plotted for the same gene sets. Gene sets with positive NES and the top 15 gene sets with highest difference between both comparisons for negative NES are plotted. (C) Heatmap for gene expression levels of 25 genes, that show a change towards wild type levels by RGFP109 treatment in R6/1 mice, from a list of genes whose expression were restored by HDACi treatment in a previous study (Thomas et al., 2008). Mean FPKM value/group is shown. Each raw is a gene and each column is a group. $n=5$ [WT-vehicle], $n=6$ [R6/1-vehicle], $n=7$ [R6/1RGFP109].

Figure 3. Effect of RGFP109 treatment on HD-associated atrophic brain substructures in R6/1 mice. Mouse brain MR images were registered to the Allen brain atlas. Volumes of substructures (absolute or normalized to whole brain volumes) were analyzed by two-sample t-test (FDR, $\mathrm{q}<0.1$ ). (A) Mean absolute volume differences of brain regions with significant volume change in R6/1 
compared to WT mice (419). (B) Mean relative volume differences of brain regions with significant volume change in R6/1 compared to WT mice (220). (A, B) Black: substructures affected positively by drug (not significant; red frame: >20\% of R6/1-WT volume difference); Gray: substructures affected negatively by drug (not significant). (C) MR images, exemplarity shown for three individuals; selected regions are color-coded. Anatomical labels are based on the Allen brain atlas.

Figure S1. Behavioral and physical assessment of vehicle- and RGFP109-treated wild type and R6/1 mice. (A) Motor performance on a RotaRod: Speed at fall as mean of all trials. (B) Open field exploration evaluating general locomotor activity measured as average velocity and vertical activity (supported rearing). (C) Elevated plus maze test showing time in open arm, path in open arm, total path, and visits and time in open arm normalized to total path. (D) Clasping score of R6/1 mice measured at the end of the study. (E) Body weight over the course of the treatment. Data represent mean+SD; n=10 [WT-vehicle], n=12(13, Open Field) [R6/1-vehicle], n=10 [WT-RGFP109], n=12 [R6/1-RGF109]; *p $\leq 0.05, * * \mathrm{p} \leq 0.01, * * * \mathrm{p} \leq 0.001, * * * * \mathrm{p} \leq 0.0001$, ns not significant by two-way ANOVA with Tukey‘s multiple comparisons test (A-C) or by unpaired Student's t-test (D).

Figure S2. Effect of HDAC1 and HDAC3 inhibition by RGFP109 on gene expression in striatum of R6/1 mice. (A) Quantitative real-time PCR analysis of genes usually up- and downregulated in $\mathrm{HD}$, motor-learning associated genes and those genes whose expression are found to be significantly altered in RNA-seq data by RGFP109 treatment of R6/1s in the striatum of vehicle- and RGFP109treated wild type (WT) and R6/1 mice. Data represent mean+SD; n=10 [WT-vehicle], n=12 [R6/1vehicle], $n=10$ [WT-RGFP109], n=12 [R6/1-RGF109]. (B) Heatmap showing differential expressed genes of R6/1 and WT comparison (FDR, $\mathrm{q}<0.1, \log 2 \mathrm{FC}>|0.5|$ ) which are changed towards WT levels by RGFP109 treatment in R6/1 mice by at least 20\% (283). For better visualization FPKM values were mean-normalized. (C) Gene Ontology (GO) analysis of genes from (B). As background, all expressed genes with FPKM $>0.1$ in at least one sample were used (18844). Ratio describes the ratio between genes in the target gene list and the total number of input genes associated with a specific GO term. Enriched GO terms with FDR, q<0.05 are plotted; BP - biological process, CC cellular component, MF - molecular function. $n=5$ [WT-vehicle], n=6 [R6/1-vehicle], n=6 [WTRFGP109], $n=7$ [R6/1-RGFP109].

Figure S3. Influence of RGFP109 treatment on brain substructures in R6/1s measured by volumetric MRI. (A, B) Heatmaps illustrating absolute (A) or relative (B) volumes of regions with significant volume difference in WT-vehicle and R6/1-vehicle comparison (419 regions for absolute and 220 regions for relative volume). Volumes of every region were normalized to the mean volume of each region over all samples. (C) Venn diagram indicating shared and unique brain regions with significant volume change in R6/1 mice from absolute and relative volume analysis. (D) Venn diagram indicating shared and unique brain regions affected positively by the drug by at least $20 \%$ 
(not-significant) in R6/1 mice (red framed regions of Figure 3A and B) from absolute and relative volume analysis. (E) Absolute volumes of selected brain regions. Data represent mean+SD; $n=10$ [WT-vehicle], $\mathrm{n}=13$ [R6/1-vehicle], $\mathrm{n}=10$ [WT-RGFP109], $\mathrm{n}=12$ [R6/1-RGFP109]; * $\mathrm{q} \leq 0.05, * * \mathrm{q} \leq$ $0.01, * * * \mathrm{q} \leq 0.001, * * * * \mathrm{q} \leq 0.0001$, ns not significant. 


\section{References}

Backman, L., Robins-Wahlin, T.B., Lundin, A., Ginovart, N., and Farde, L. (1997). Cognitive deficits in Huntington's disease are predicted by dopaminergic PET markers and brain volumes. Brain 120 ( Pt 12), 2207-2217.

Badea, A., Ng, K.L., Anderson, R.J., Zhang, J., Miller, M.I., and O'Brien, R.J. (2019). Magnetic resonance imaging of mouse brain networks plasticity following motor learning. PLoS One 14, e0216596.

Bayram-Weston, Z., Jones, L., Dunnett, S.B., and Brooks, S.P. (2012). Light and electron microscopic characterization of the evolution of cellular pathology in the R6/1 Huntington's disease transgenic mice. Brain Res Bull 88, 104-112.

Benn, C.L., Sun, T., Sadri-Vakili, G., McFarland, K.N., DiRocco, D.P., Yohrling, G.J., Clark, T.W., Bouzou, B., and Cha, J.H. (2008). Huntingtin modulates transcription, occupies gene promoters in vivo, and binds directly to DNA in a polyglutamine-dependent manner. J Neurosci 28, 10720-10733.

Bett, J.S., Benn, C.L., Ryu, K.Y., Kopito, R.R., and Bates, G.P. (2009). The polyubiquitin Ubc gene modulates histone $\mathrm{H} 2 \mathrm{~A}$ monoubiquitylation in the R6/2 mouse model of Huntington's disease. J Cell Mol Med 13, 2645-2657.

Bissonnette, S., Vaillancourt, M., Hebert, S.S., Drolet, G., and Samadi, P. (2013). Striatal preenkephalin overexpression improves Huntington's disease symptoms in the R6/2 mouse model of Huntington's disease. PLoS One 8, e75099.

Brooks, S.P., Janghra, N., Workman, V.L., Bayram-Weston, Z., Jones, L., and Dunnett, S.B. (2012). Longitudinal analysis of the behavioural phenotype in R6/1 (C57BL/6J) Huntington's disease transgenic mice. Brain Res Bull 88, 94-103.

Buitrago, M.M., Schulz, J.B., Dichgans, J., and Luft, A.R. (2004). Short and long-term motor skill learning in an accelerated rotarod training paradigm. Neurobiol Learn Mem 81, 211-216.

Butler, R., and Bates, G.P. (2006). Histone deacetylase inhibitors as therapeutics for polyglutamine disorders. Nat Rev Neurosci 7, 784-796.

Cepeda, C., Murphy, K.P., Parent, M., and Levine, M.S. (2014). The role of dopamine in Huntington's disease. Prog Brain Res 211, 235-254.

Cha, J.H. (2000). Transcriptional dysregulation in Huntington's disease. Trends Neurosci 23, 387-392.

Chen, J.Y., Wang, E., Galvan, L., Huynh, M., Joshi, P., Cepeda, C., and Levine, M.S. (2013). Effects of the Pimelic Diphenylamide Histone Deacetylase Inhibitor HDACi 4b on the R6/2 and N171-82Q Mouse Models of Huntington's Disease. PLoS Curr 5.

Chiu, C.T., Liu, G., Leeds, P., and Chuang, D.M. (2011). Combined treatment with the mood stabilizers lithium and valproate produces multiple beneficial effects in transgenic mouse models of Huntington's disease. Neuropsychopharmacology 36, 2406-2421.

Cong, S.Y., Pepers, B.A., Evert, B.O., Rubinsztein, D.C., Roos, R.A., van Ommen, G.J., and Dorsman, J.C. (2005). Mutant huntingtin represses CBP, but not p300, by binding and protein degradation. Mol Cell Neurosci 30, 560-571.

Coppen, E.M., and Roos, R.A. (2017). Current Pharmacological Approaches to Reduce Chorea in Huntington's Disease. Drugs 77, 29-46. 
Cybulska-Klosowicz, A., Mazarakis, N.K., Van Dellen, A., Blakemore, C., Hannan, A.J., and Kossut, M. (2004). Impaired learning-dependent cortical plasticity in Huntington's disease transgenic mice. Neurobiol Dis 17, 427-434.

D'Amours, G., Bureau, G., Boily, M.J., and Cyr, M. (2011). Differential gene expression profiling in the mouse brain during motor skill learning: focus on the striatum structure. Behav Brain Res 221, $108-117$.

Duyao, M., Ambrose, C., Myers, R., Novelletto, A., Persichetti, F., Frontali, M., Folstein, S., Ross, C., Franz, M., Abbott, M., et al. (1993). Trinucleotide repeat length instability and age of onset in Huntington's disease. Nat Genet 4, 387-392.

Eden, E., Navon, R., Steinfeld, I., Lipson, D., and Yakhini, Z. (2009). GOrilla: a tool for discovery and visualization of enriched GO terms in ranked gene lists. BMC Bioinformatics 10, 48.

Faraco, G., Pancani, T., Formentini, L., Mascagni, P., Fossati, G., Leoni, F., Moroni, F., and Chiarugi, A. (2006). Pharmacological inhibition of histone deacetylases by suberoylanilide hydroxamic acid specifically alters gene expression and reduces ischemic injury in the mouse brain. Mol Pharmacol 70, 1876-1884.

Ferrante, R.J., Kubilus, J.K., Lee, J., Ryu, H., Beesen, A., Zucker, B., Smith, K., Kowall, N.W., Ratan, R.R., Luthi-Carter, R., et al. (2003). Histone deacetylase inhibition by sodium butyrate chemotherapy ameliorates the neurodegenerative phenotype in Huntington's disease mice. J Neurosci 23, 9418-9427.

Ferrante, R.J., Ryu, H., Kubilus, J.K., D'Mello, S., Sugars, K.L., Lee, J., Lu, P., Smith, K., Browne, S., Beal, M.F., et al. (2004). Chemotherapy for the brain: the antitumor antibiotic mithramycin prolongs survival in a mouse model of Huntington's disease. J Neurosci 24, 10335-10342.

Harrison, I.F., and Dexter, D.T. (2013). Epigenetic targeting of histone deacetylase: therapeutic potential in Parkinson's disease? Pharmacol Ther 140, 34-52.

Hervas-Corpion, I., Guiretti, D., Alcaraz-Iborra, M., Olivares, R., Campos-Caro, A., Barco, A., and Valor, L.M. (2018). Early alteration of epigenetic-related transcription in Huntington's disease mouse models. Sci Rep 8, 9925.

Hockly, E., Richon, V.M., Woodman, B., Smith, D.L., Zhou, X., Rosa, E., Sathasivam, K., GhaziNoori, S., Mahal, A., Lowden, P.A., et al. (2003). Suberoylanilide hydroxamic acid, a histone deacetylase inhibitor, ameliorates motor deficits in a mouse model of Huntington's disease. Proc Natl Acad Sci U S A 100, 2041-2046.

Hodges, A., Hughes, G., Brooks, S., Elliston, L., Holmans, P., Dunnett, S.B., and Jones, L. (2008). Brain gene expression correlates with changes in behavior in the R6/1 mouse model of Huntington's disease. Genes Brain Behav 7, 288-299.

Hodges, A., Strand, A.D., Aragaki, A.K., Kuhn, A., Sengstag, T., Hughes, G., Elliston, L.A., Hartog, C., Goldstein, D.R., Thu, D., et al. (2006). Regional and cellular gene expression changes in human Huntington's disease brain. Hum Mol Genet 15, 965-977.

Jia, H., Pallos, J., Jacques, V., Lau, A., Tang, B., Cooper, A., Syed, A., Purcell, J., Chen, Y., Sharma, S., et al. (2012). Histone deacetylase (HDAC) inhibitors targeting HDAC3 and HDAC1 ameliorate polyglutamine-elicited phenotypes in model systems of Huntington's disease. Neurobiol Dis 46, 351361. 
Jia, H., Wang, Y., Morris, C.D., Jacques, V., Gottesfeld, J.M., Rusche, J.R., and Thomas, E.A. (2016). The Effects of Pharmacological Inhibition of Histone Deacetylase 3 (HDAC3) in Huntington's Disease Mice. PLoS One 11, e0152498.

Jiang, H., Poirier, M.A., Liang, Y., Pei, Z., Weiskittel, C.E., Smith, W.W., DeFranco, D.B., and Ross, C.A. (2006). Depletion of CBP is directly linked with cellular toxicity caused by mutant huntingtin. Neurobiol Dis 23, 543-551.

Johnston, T.H., Huot, P., Damude, S., Fox, S.H., Jones, S.W., Rusche, J.R., and Brotchie, J.M. (2013). RGFP109, a histone deacetylase inhibitor attenuates L-DOPA-induced dyskinesia in the MPTP-lesioned marmoset: a proof-of-concept study. Parkinsonism Relat Disord 19, 260-264.

Kazantsev, A.G., and Thompson, L.M. (2008). Therapeutic application of histone deacetylase inhibitors for central nervous system disorders. Nat Rev Drug Discov 7, 854-868.

Koch, S., Mueller, S., Foddis, M., Bienert, T., von Elverfeldt, D., Knab, F., Farr, T.D., Bernard, R., Dopatka, M., Rex, A., et al. (2017). Atlas registration for edema-corrected MRI lesion volume in mouse stroke models. J Cereb Blood Flow Metab, 271678X17726635.

Koch, S., Mueller, S., Foddis, M., Bienert, T., von Elverfeldt, D., Knab, F., Farr, T.D., Bernard, R., Dopatka, M., Rex, A., et al. (2019). Atlas registration for edema-corrected MRI lesion volume in mouse stroke models. J Cereb Blood Flow Metab 39, 313-323.

Kurdistani, S.K., Tavazoie, S., and Grunstein, M. (2004). Mapping global histone acetylation patterns to gene expression. Cell 117, 721-733.

Lawrence, A.D., Watkins, L.H., Sahakian, B.J., Hodges, J.R., and Robbins, T.W. (2000). Visual object and visuospatial cognition in Huntington's disease: implications for information processing in corticostriatal circuits. Brain 123 ( Pt 7), 1349-1364.

Lein, E.S., Hawrylycz, M.J., Ao, N., Ayres, M., Bensinger, A., Bernard, A., Boe, A.F., Boguski, M.S., Brockway, K.S., Byrnes, E.J., et al. (2007). Genome-wide atlas of gene expression in the adult mouse brain. Nature 445, 168-176.

Lim, S., Chesser, A.S., Grima, J.C., Rappold, P.M., Blum, D., Przedborski, S., and Tieu, K. (2011). D-beta-hydroxybutyrate is protective in mouse models of Huntington's disease. PLoS One 6, e24620.

Mangiarini, L., Sathasivam, K., Seller, M., Cozens, B., Harper, A., Hetherington, C., Lawton, M., Trottier, Y., Lehrach, H., Davies, S.W., et al. (1996). Exon 1 of the HD gene with an expanded CAG repeat is sufficient to cause a progressive neurological phenotype in transgenic mice. Cell 87, 493506.

Mazarakis, N.K., Cybulska-Klosowicz, A., Grote, H., Pang, T., Van Dellen, A., Kossut, M., Blakemore, C., and Hannan, A.J. (2005). Deficits in experience-dependent cortical plasticity and sensory-discrimination learning in presymptomatic Huntington's disease mice. J Neurosci 25, 30593066.

Meisel, A., Harms, C., Yildirim, F., Bosel, J., Kronenberg, G., Harms, U., Fink, K.B., and Endres, M. (2006). Inhibition of histone deacetylation protects wild-type but not gelsolin-deficient neurons from oxygen/glucose deprivation. J Neurochem 98, 1019-1031.

Mootha, V.K., Lindgren, C.M., Eriksson, K.F., Subramanian, A., Sihag, S., Lehar, J., Puigserver, P., Carlsson, E., Ridderstrale, M., Laurila, E., et al. (2003). PGC-1alpha-responsive genes involved in oxidative phosphorylation are coordinately downregulated in human diabetes. Nat Genet 34, 267-273. 
Naver, B., Stub, C., Moller, M., Fenger, K., Hansen, A.K., Hasholt, L., and Sorensen, S.A. (2003). Molecular and behavioral analysis of the R6/1 Huntington's disease transgenic mouse. Neuroscience $122,1049-1057$.

Nucifora, F.C., Jr., Sasaki, M., Peters, M.F., Huang, H., Cooper, J.K., Yamada, M., Takahashi, H., Tsuji, S., Troncoso, J., Dawson, V.L., et al. (2001). Interference by huntingtin and atrophin-1 with cbp-mediated transcription leading to cellular toxicity. Science 291, 2423-2428.

Pallos, J., Bodai, L., Lukacsovich, T., Purcell, J.M., Steffan, J.S., Thompson, L.M., and Marsh, J.L. (2008). Inhibition of specific HDACs and sirtuins suppresses pathogenesis in a Drosophila model of Huntington's disease. Hum Mol Genet 17, 3767-3775.

Rai, M., Soragni, E., Chou, C.J., Barnes, G., Jones, S., Rusche, J.R., Gottesfeld, J.M., and Pandolfo, M. (2010). Two new pimelic diphenylamide HDAC inhibitors induce sustained frataxin upregulation in cells from Friedreich's ataxia patients and in a mouse model. PLoS One 5, e8825.

Rangel-Barajas, C., Coronel, I., and Floran, B. (2015). Dopamine Receptors and Neurodegeneration. Aging Dis 6, 349-368.

Rattray, I., Smith, E.J., Crum, W.R., Walker, T.A., Gale, R., Bates, G.P., and Modo, M. (2013). Correlations of behavioral deficits with brain pathology assessed through longitudinal MRI and histopathology in the R6/1 mouse model of Huntington's disease. PLoS One 8, e84726.

Rosas, H.D., Koroshetz, W.J., Chen, Y.I., Skeuse, C., Vangel, M., Cudkowicz, M.E., Caplan, K., Marek, K., Seidman, L.J., Makris, N., et al. (2003). Evidence for more widespread cerebral pathology in early HD: an MRI-based morphometric analysis. Neurology 60, 1615-1620.

Ryu, H., Lee, J., Hagerty, S.W., Soh, B.Y., McAlpin, S.E., Cormier, K.A., Smith, K.M., and Ferrante, R.J. (2006). ESET/SETDB1 gene expression and histone H3 (K9) trimethylation in Huntington's disease. Proc Natl Acad Sci U S A 103, 19176-19181.

Ryu, H., Smith, K., Camelo, S.I., Carreras, I., Lee, J., Iglesias, A.H., Dangond, F., Cormier, K.A., Cudkowicz, M.E., Brown, R.H., Jr., et al. (2005). Sodium phenylbutyrate prolongs survival and regulates expression of anti-apoptotic genes in transgenic amyotrophic lateral sclerosis mice. $\mathrm{J}$ Neurochem 93, 1087-1098.

Sadri-Vakili, G., Bouzou, B., Benn, C.L., Kim, M.O., Chawla, P., Overland, R.P., Glajch, K.E., Xia, E., Qiu, Z., Hersch, S.M., et al. (2007). Histones associated with downregulated genes are hypoacetylated in Huntington's disease models. Hum Mol Genet 16, 1293-1306.

Sandi, C., Pinto, R.M., Al-Mahdawi, S., Ezzatizadeh, V., Barnes, G., Jones, S., Rusche, J.R., Gottesfeld, J.M., and Pook, M.A. (2011). Prolonged treatment with pimelic o-aminobenzamide HDAC inhibitors ameliorates the disease phenotype of a Friedreich ataxia mouse model. Neurobiol Dis 42, 496-505.

Scholz, J., Niibori, Y., P, W.F., and J, P.L. (2015). Rotarod training in mice is associated with changes in brain structure observable with multimodal MRI. Neuroimage 107, 182-189.

Schweizer, S., Harms, C., Lerch, H., Flynn, J., Hecht, J., Yildirim, F., Meisel, A., and Marschenz, S. (2015). Inhibition of histone methyltransferases SUV39H1 and G9a leads to neuroprotection in an in vitro model of cerebral ischemia. J Cereb Blood Flow Metab 35, 1640-1647.

Seo, H., Kim, W., and Isacson, O. (2008). Compensatory changes in the ubiquitin-proteasome system, brain-derived neurotrophic factor and mitochondrial complex II/III in YAC72 and R6/2 transgenic mice partially model Huntington's disease patients. Hum Mol Genet 17, 3144-3153. 
Seredenina, T., and Luthi-Carter, R. (2012). What have we learned from gene expression profiles in Huntington's disease? Neurobiol Dis 45, 83-98.

Soragni, E., Miao, W., Iudicello, M., Jacoby, D., De Mercanti, S., Clerico, M., Longo, F., Piga, A., Ku, S., Campau, E., et al. (2014). Epigenetic therapy for Friedreich ataxia. Ann Neurol 76, 489-508.

Steffan, J.S., Bodai, L., Pallos, J., Poelman, M., McCampbell, A., Apostol, B.L., Kazantsev, A., Schmidt, E., Zhu, Y.Z., Greenwald, M., et al. (2001). Histone deacetylase inhibitors arrest polyglutamine-dependent neurodegeneration in Drosophila. Nature 413, 739-743.

Steffan, J.S., Kazantsev, A., Spasic-Boskovic, O., Greenwald, M., Zhu, Y.Z., Gohler, H., Wanker, E.E., Bates, G.P., Housman, D.E., and Thompson, L.M. (2000). The Huntington's disease protein interacts with p53 and CREB-binding protein and represses transcription. Proc Natl Acad Sci U S A 97, 6763-6768.

Subramanian, A., Tamayo, P., Mootha, V.K., Mukherjee, S., Ebert, B.L., Gillette, M.A., Paulovich, A., Pomeroy, S.L., Golub, T.R., Lander, E.S., et al. (2005). Gene set enrichment analysis: a knowledge-based approach for interpreting genome-wide expression profiles. Proc Natl Acad Sci U S A $102,15545-15550$.

Suelves, N., Kirkham-McCarthy, L., Lahue, R.S., and Gines, S. (2017). A selective inhibitor of histone deacetylase 3 prevents cognitive deficits and suppresses striatal CAG repeat expansions in Huntington's disease mice. Sci Rep 7, 6082.

Tabrizi, S.J., Reilmann, R., Roos, R.A., Durr, A., Leavitt, B., Owen, G., Jones, R., Johnson, H., Craufurd, D., Hicks, S.L., et al. (2012). Potential endpoints for clinical trials in premanifest and early Huntington's disease in the TRACK-HD study: analysis of 24 month observational data. Lancet Neurol 11, 42-53.

Tamakoshi, K., Ishida, A., Takamatsu, Y., Hamakawa, M., Nakashima, H., Shimada, H., and Ishida, K. (2014). Motor skills training promotes motor functional recovery and induces synaptogenesis in the motor cortex and striatum after intracerebral hemorrhage in rats. Behav Brain Res 260, 34-43.

The Huntington's Disease Collaborative Research Group (1993). A novel gene containing a trinucleotide repeat that is expanded and unstable on Huntington's disease chromosomes. The Huntington's Disease Collaborative Research Group. Cell 72, 971-983.

Thomas, E.A., Coppola, G., Desplats, P.A., Tang, B., Soragni, E., Burnett, R., Gao, F., Fitzgerald, K.M., Borok, J.F., Herman, D., et al. (2008). The HDAC inhibitor $4 \mathrm{~b}$ ameliorates the disease phenotype and transcriptional abnormalities in Huntington's disease transgenic mice. Proc Natl Acad Sci U S A 105, 15564-15569.

Vashishtha, M., Ng, C.W., Yildirim, F., Gipson, T.A., Kratter, I.H., Bodai, L., Song, W., Lau, A., Labadorf, A., Vogel-Ciernia, A., et al. (2013). Targeting H3K4 trimethylation in Huntington disease. Proc Natl Acad Sci U S A 110, E3027-3036.

Wagner, F.F., Zhang, Y.L., Fass, D.M., Joseph, N., Gale, J.P., Weiwer, M., McCarren, P., Fisher, S.L., Kaya, T., Zhao, W.N., et al. (2015). Kinetically Selective Inhibitors of Histone Deacetylase 2 (HDAC2) as Cognition Enhancers. Chem Sci 6, 804-815.

Walf, A.A., and Frye, C.A. (2007). The use of the elevated plus maze as an assay of anxiety-related behavior in rodents. Nat Protoc 2, 322-328. 
Wang, Z., Zang, C., Rosenfeld, J.A., Schones, D.E., Barski, A., Cuddapah, S., Cui, K., Roh, T.Y., Peng, W., Zhang, M.Q., et al. (2008). Combinatorial patterns of histone acetylations and methylations in the human genome. Nat Genet 40, 897-903.

Yang, S.S., Zhang, R., Wang, G., and Zhang, Y.F. (2017). The development prospection of HDAC inhibitors as a potential therapeutic direction in Alzheimer's disease. Transl Neurodegener 6, 19.

Yildirim, F., Gertz, K., Kronenberg, G., Harms, C., Fink, K.B., Meisel, A., and Endres, M. (2008). Inhibition of histone deacetylation protects wildtype but not gelsolin-deficient mice from ischemic brain injury. Exp Neurol 210, 531-542.

Yildirim, F., Ng, C.W., Kappes, V., Ehrenberger, T., Rigby, S.K., Stivanello, V., Gipson, T.A., Soltis, A.R., Vanhoutte, P., Caboche, J., et al. (2019). Early epigenomic and transcriptional changes reveal Elk-1 transcription factor as a therapeutic target in Huntington's disease. Proc Natl Acad Sci U S A $116,24840-24851$.

Zamboni, V., Armentano, M., Berto, G., Ciraolo, E., Ghigo, A., Garzotto, D., Umbach, A., DiCunto, F., Parmigiani, E., Boido, M., et al. (2018). Hyperactivity of Rac1-GTPase pathway impairs neuritogenesis of cortical neurons by altering actin dynamics. Sci Rep 8, 7254.

Zuccato, C., Liber, D., Ramos, C., Tarditi, A., Rigamonti, D., Tartari, M., Valenza, M., and Cattaneo, E. (2005). Progressive loss of BDNF in a mouse model of Huntington's disease and rescue by BDNF delivery. Pharmacol Res 52, 133-139.

Zuccato, C., Marullo, M., Vitali, B., Tarditi, A., Mariotti, C., Valenza, M., Lahiri, N., Wild, E.J., Sassone, J., Ciammola, A., et al. (2011). Brain-derived neurotrophic factor in patients with Huntington's disease. PLoS One 6, e22966. 
Figure1

bjAxiv preprint doi: https://doi.org/10.1101/2020.10.13.337154; this version posted October 13, 2020. The copyright holder for this preprint

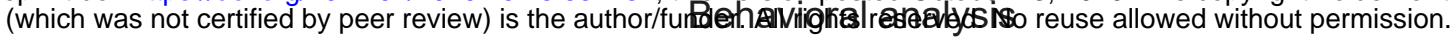

\section{RotaRod}

Open field Organ dissection

Elevated plus maze Transcriptome analysis

male WT or R6/1 mice

(11-14 weeks of age)

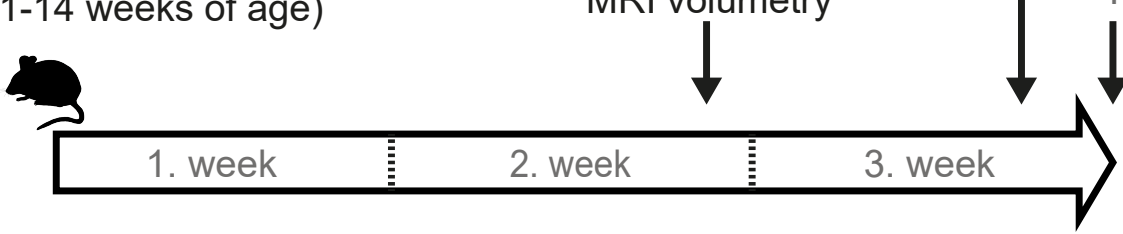

Immunohistochemistry

RGFP109 (30 mg/kg) or vehicle treatment

(i.p. injections; 5 times a week)

\section{- WT - vehicle WT - RGFP109 \\ - R6/1 - vehicle $\square$ R6/1 - RGFP109}

B

RotaRod
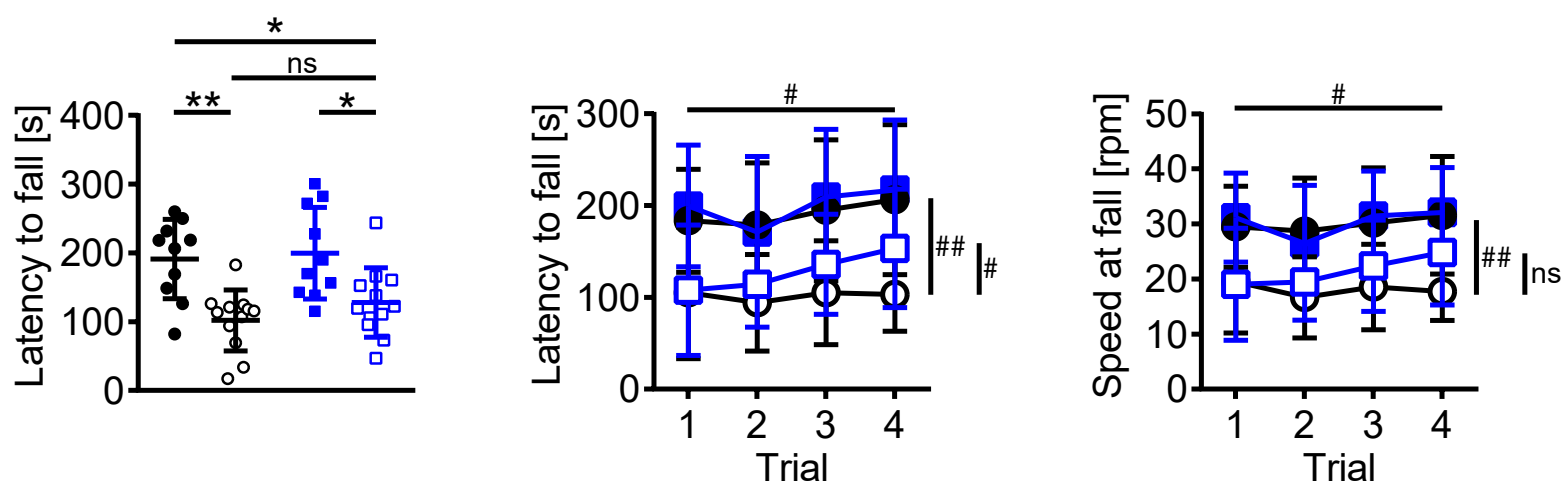

C

Open Field

D

Elevated plus maze
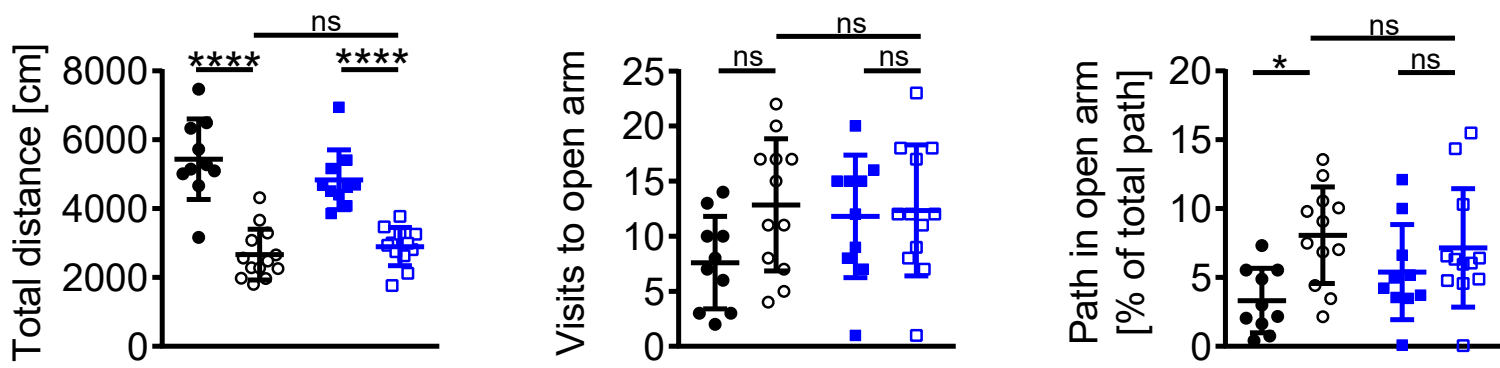
Figure2

bioRxiv preprint doi: https://doi.org/10.1101/2020.10.13.337154; this version posted October 13, 2020. The copyright holder for this preprint

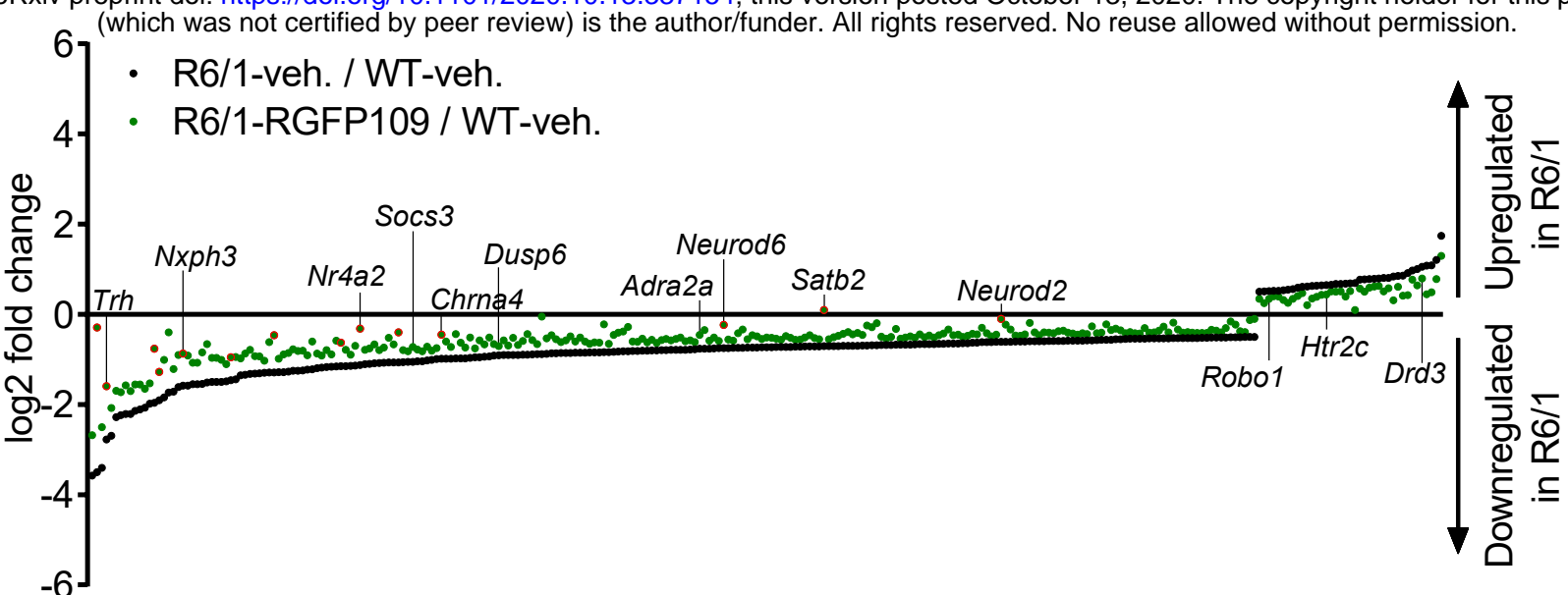

B

- NES [R6/1-vehicle / WT-vehicle]

nES [R6/1-RGFP109 / WT-vehicle]

GO_B cell proliferation

REACTOME_Growth hormone receptor signaling GO_Regulation of catecholamine secretion GO_Neuropeptide receptor binding -

GO Regulation of potassium ion transportGO_Neuropeptide hormone activity GO_Positive regulation of behaviorGO_Myosin filament

GO_Negative regulation of hormone secretion

GO_Trabecula formation GO_Regulation of amine transport REACTOME_Regulation of IFNa signaling. REACTOME_G alpha s signalling eventsKEGG_Linoleic acid metabolism GO_Protein DNA complex GO_Nuclear nucleosomeGO_DNA packaging complex-

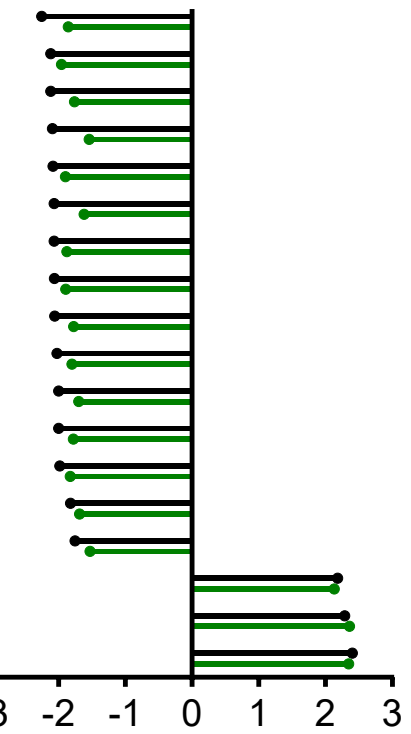

Normalized Enrichment Score

C (NES)

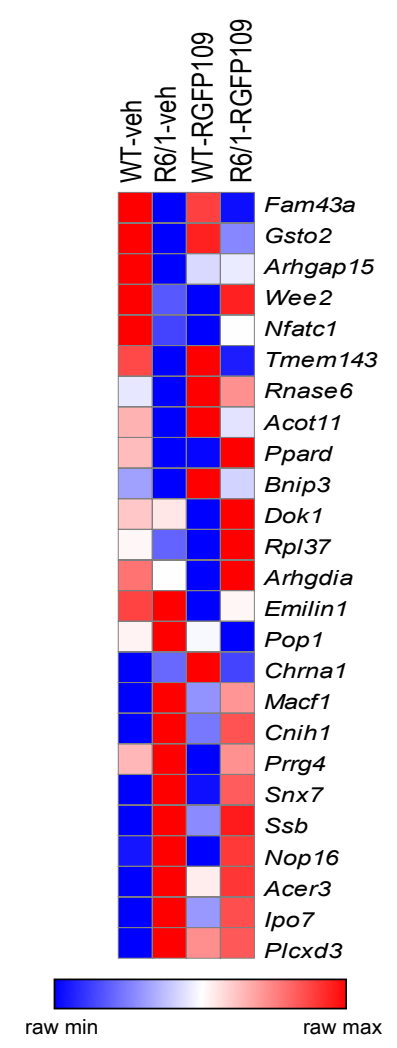


Figure 3

bioRxiv preprint doi: https://doi.org/10.1101/2020.10.13.337154; this version posted October 13, 2020. The copyright holder for this preprint

(which was not certified by peer review) is the author/funder. All rights reserved. No reuse allowed without permission.

A

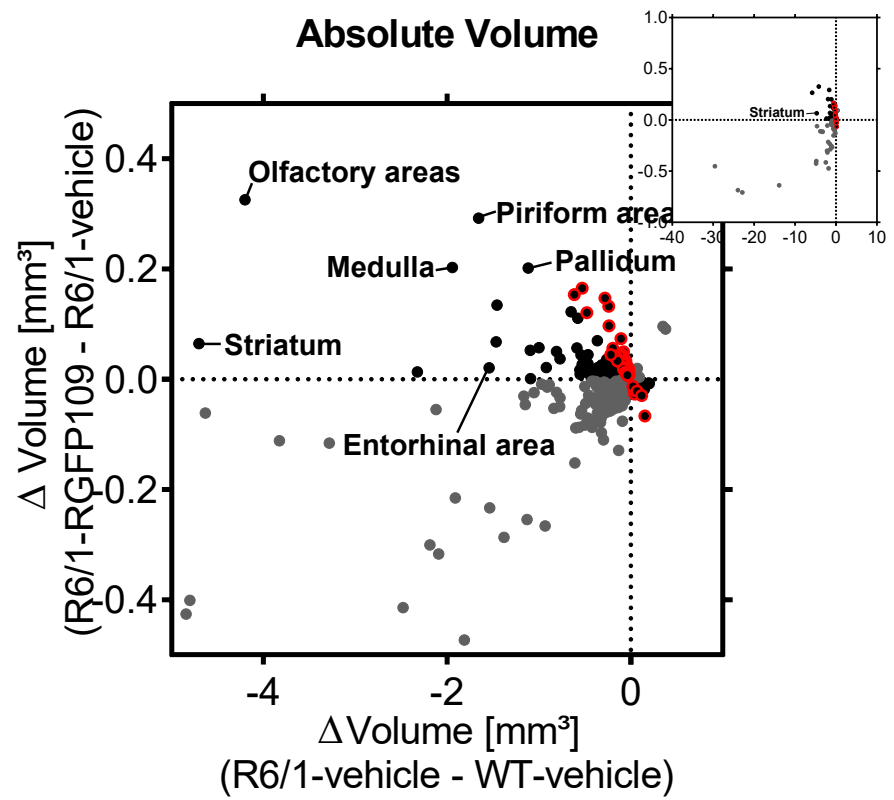

B

Relative volume

(normalized to whole brain volume)

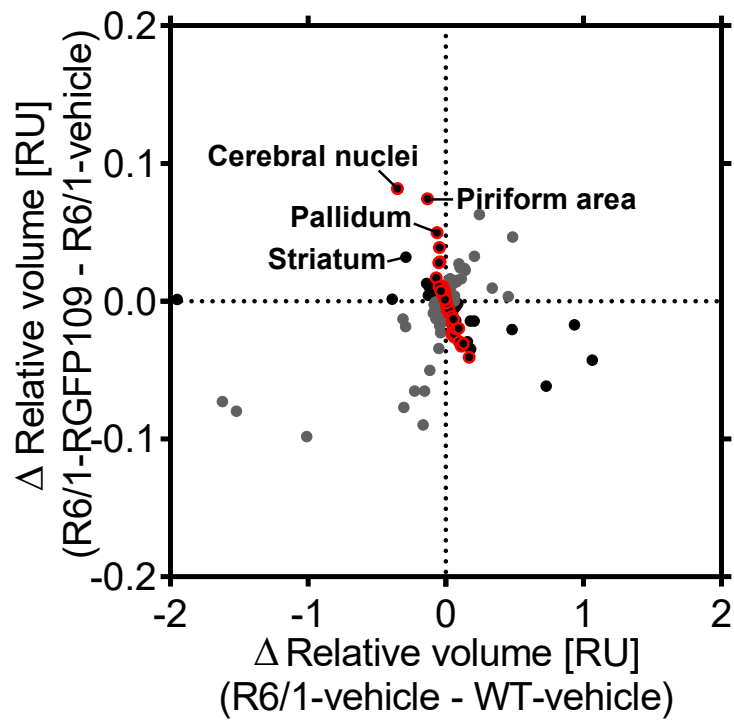

C
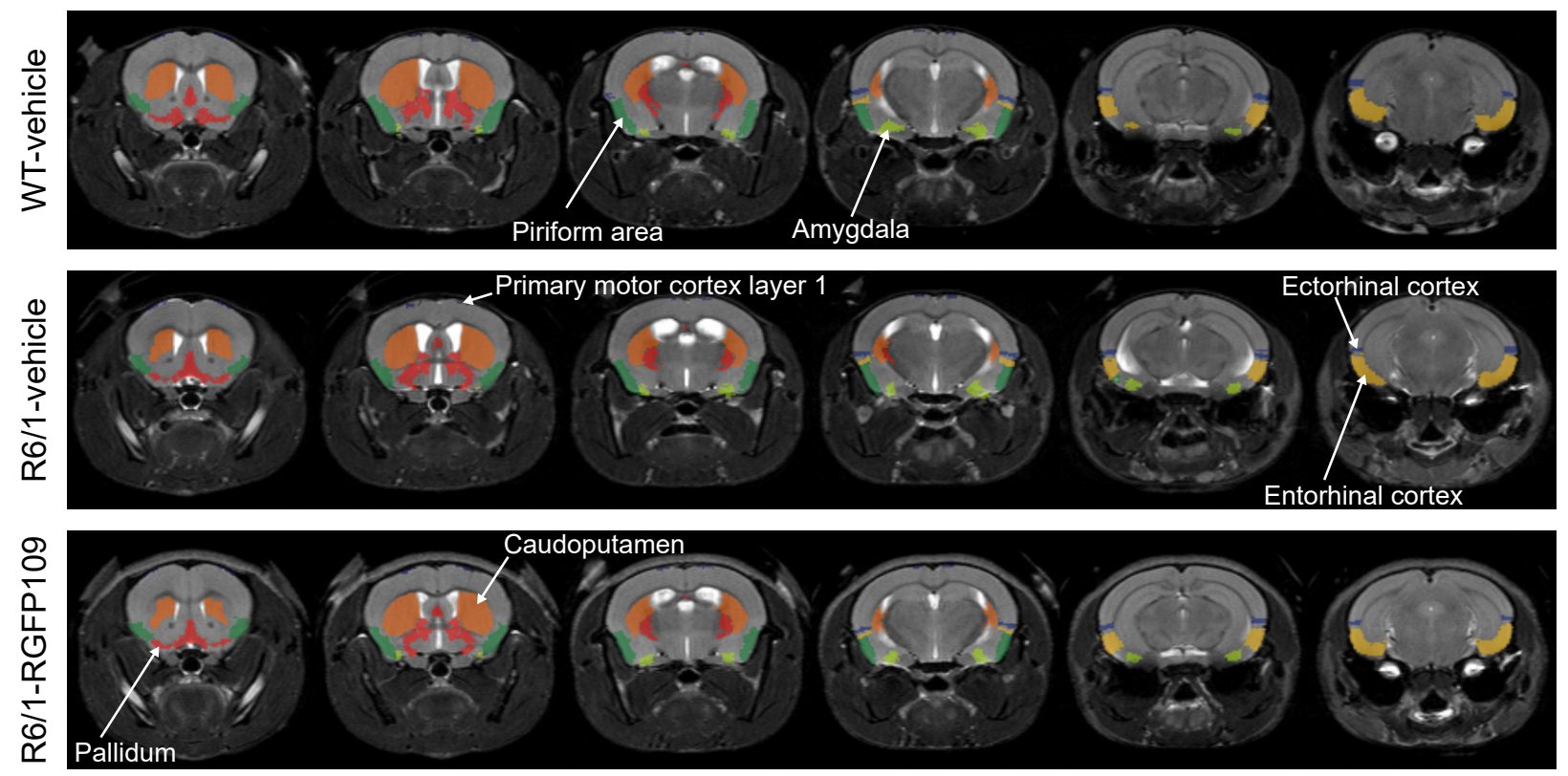

Amygdala Caudoputamen Ectorhinal cortex Entorhinal cortex Pallidum Piriform area Primary motor cortex layer 1 
A

Rotarod

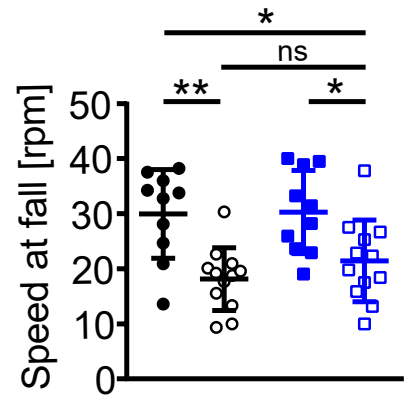

C

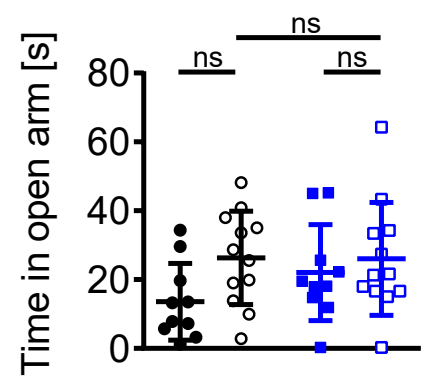

B

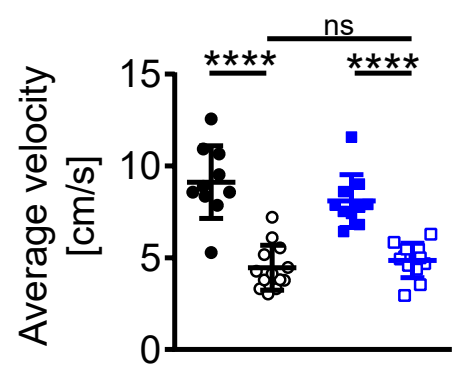

Elevated plus maze

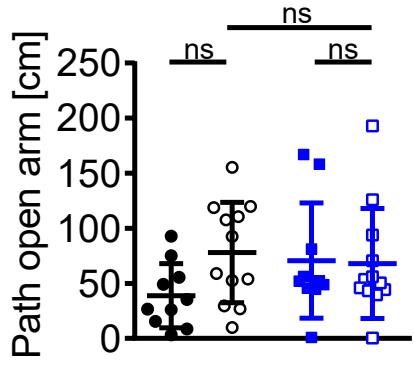

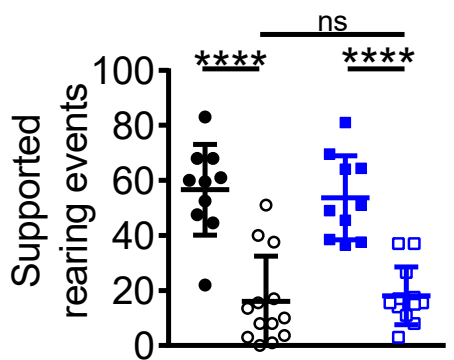

Open Field

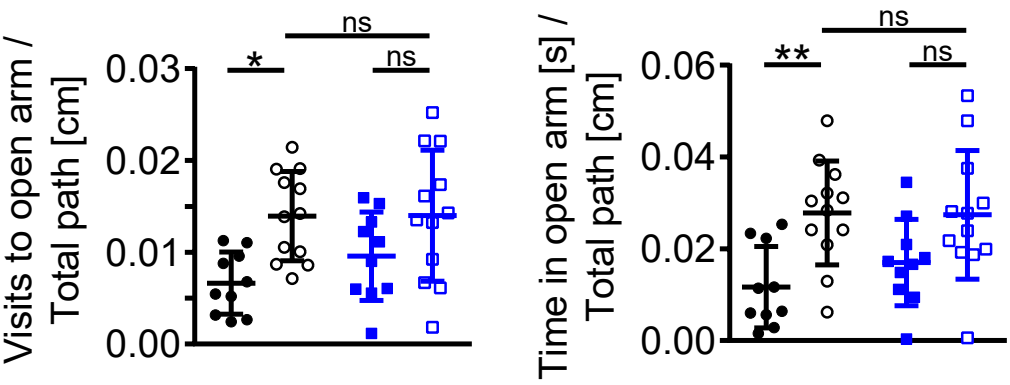

D

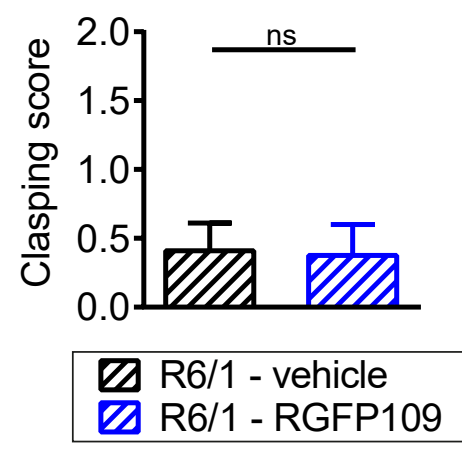

E

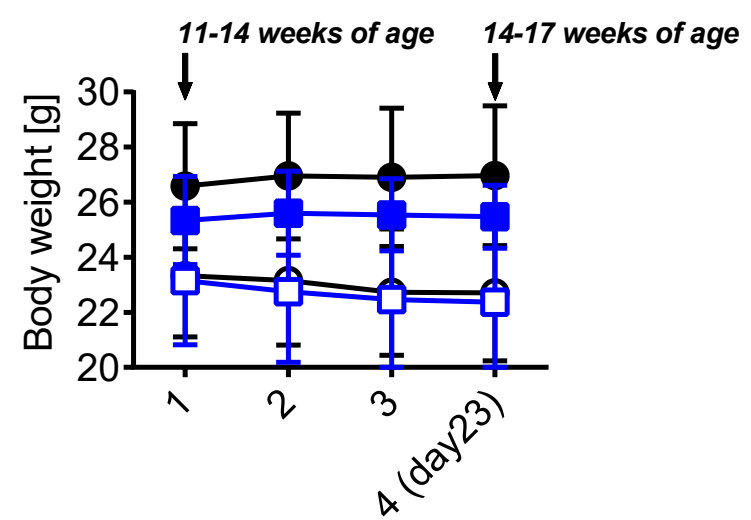

Weeks of treatment 
Figure S2

A bioRxiv preprint doi: https://doi.org/10.1101/2020.10.13.337154; this version posted October 13, 2020. The copyright holder for this preprint (which was not certified by peer review) is the author/Bnder. All rights reserved. No reuse allowed without permission.

vehicle

RGFP109
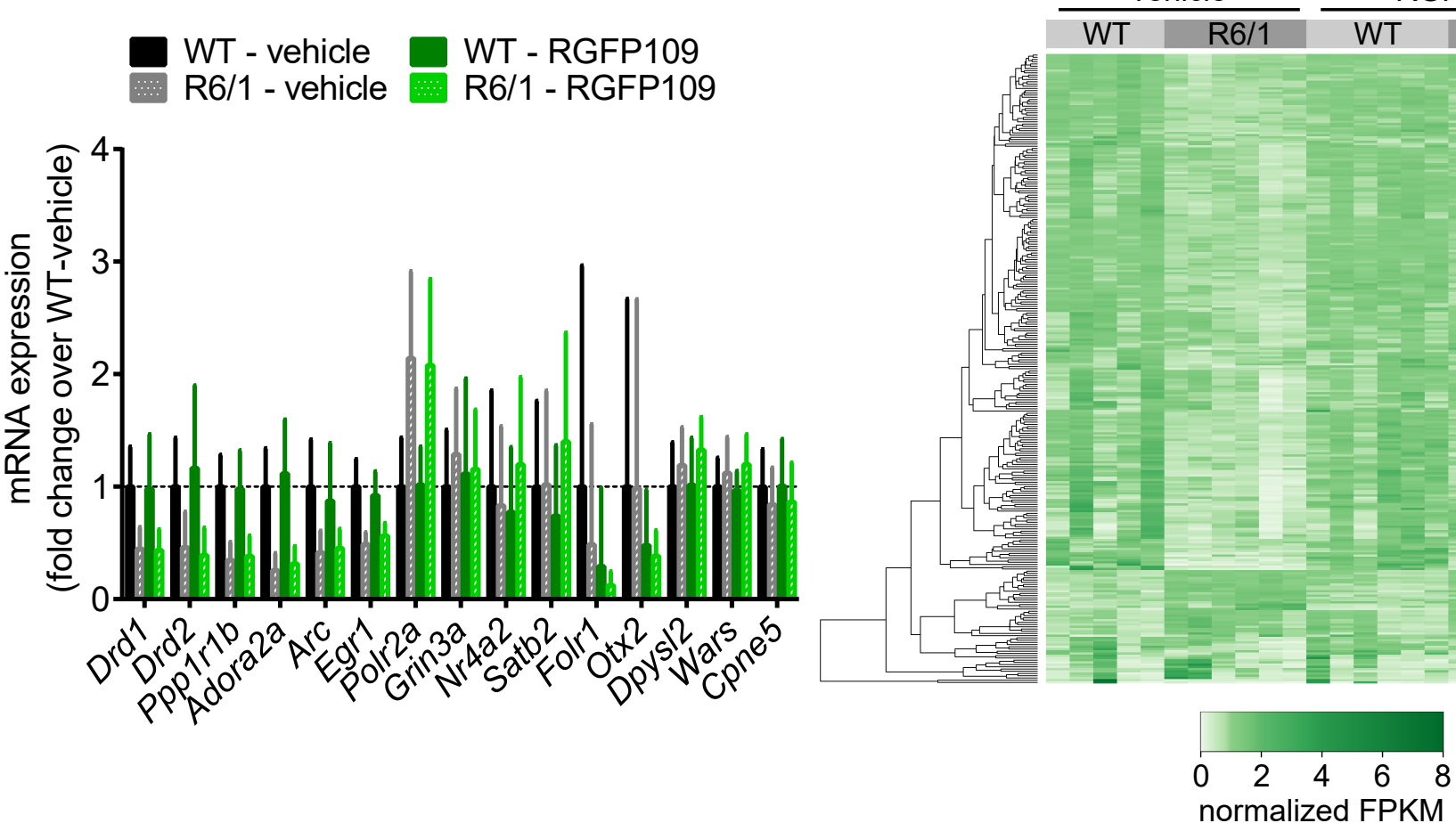

C

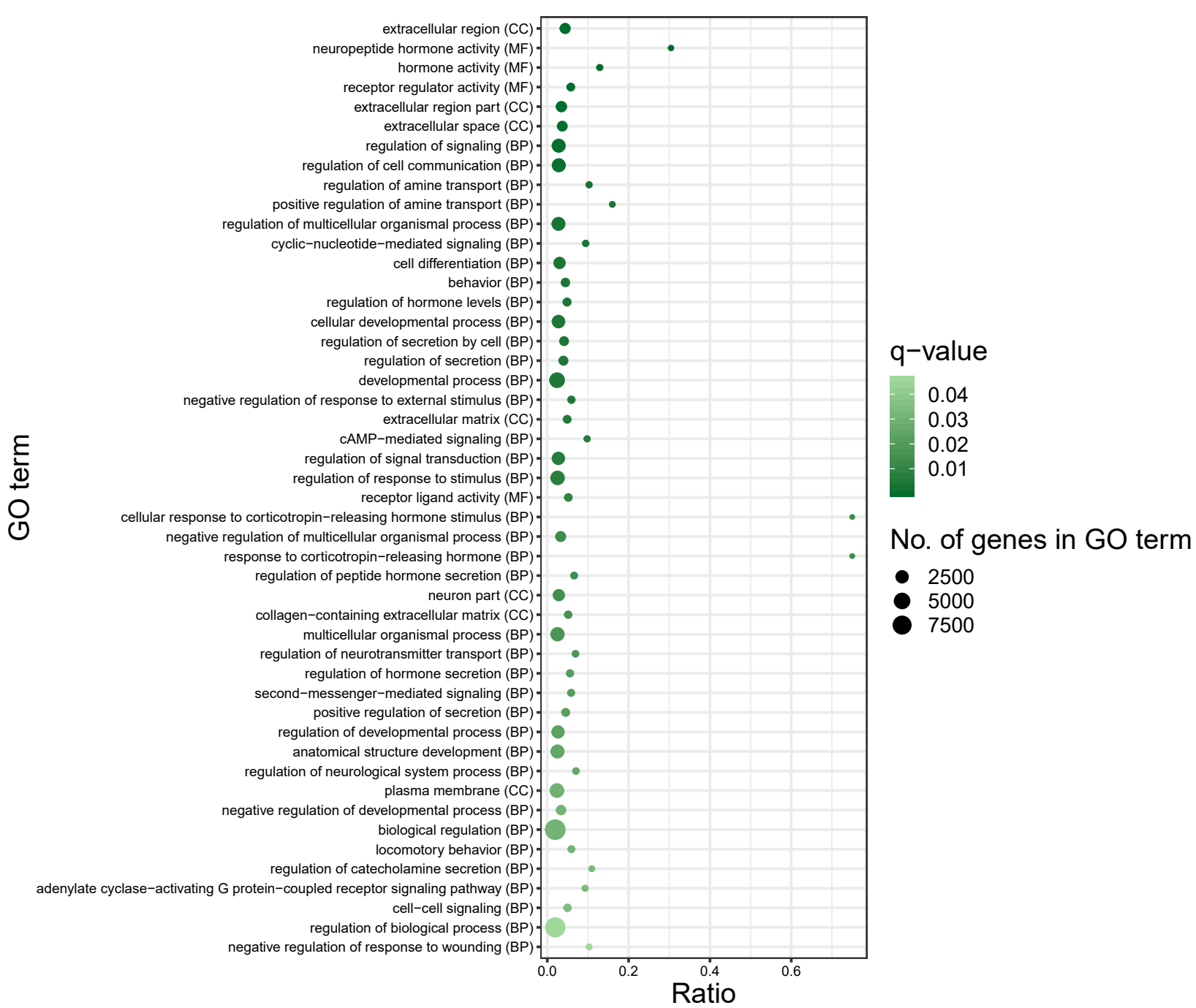


Figure S3

bioRxiv preprint doi: https://doi.org/10.1101/2020.10.13.337154; this version posted October 13, 2020. The copyright holder for this preprint

A

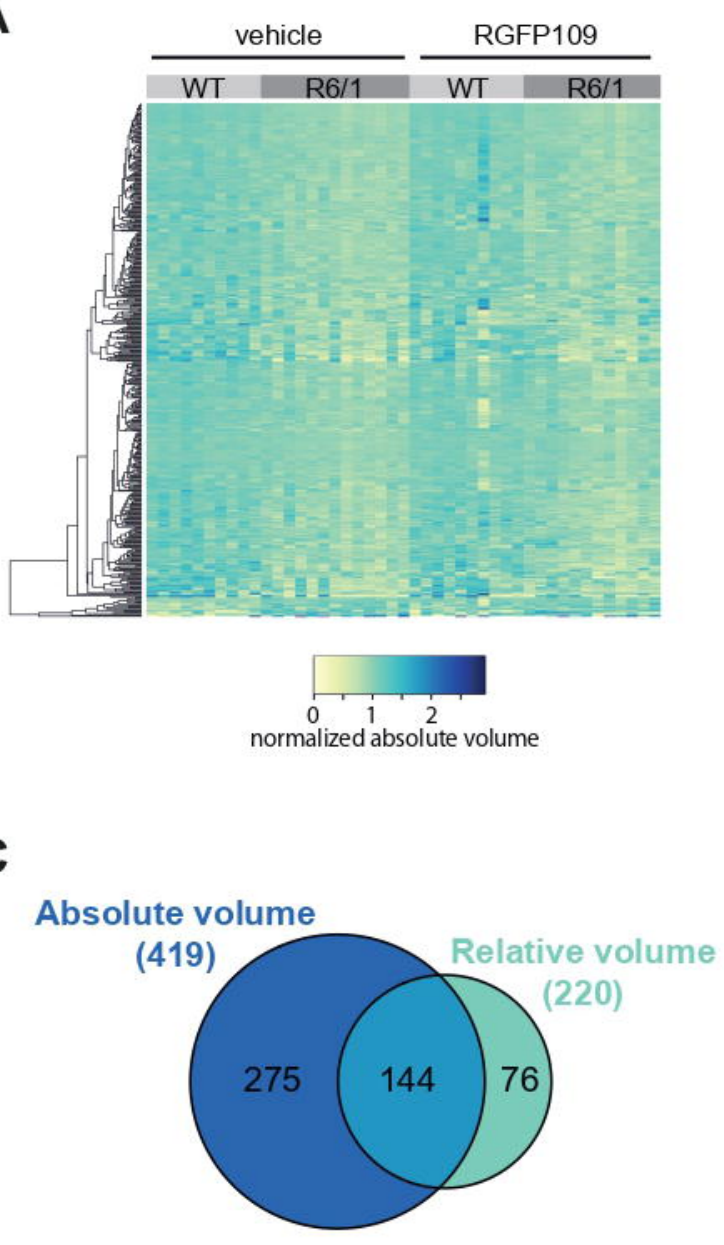

D

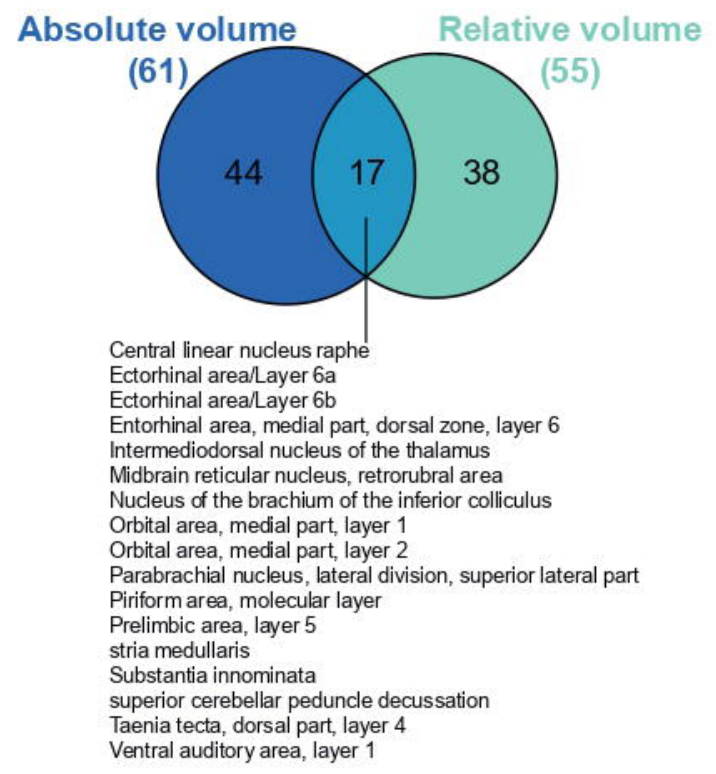

B

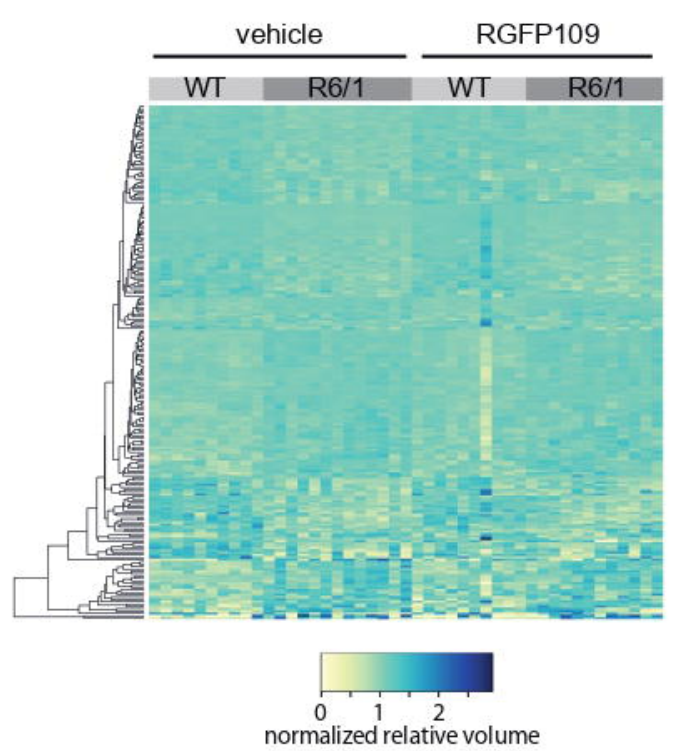

E

\begin{tabular}{|ll|}
\hline WT - vehicle & 葍 R6/1 - vehicle \\
WT - RGFP109 & 葍 R6/1 - RGFP109 \\
\hline
\end{tabular}

Whole brain volume Striatum
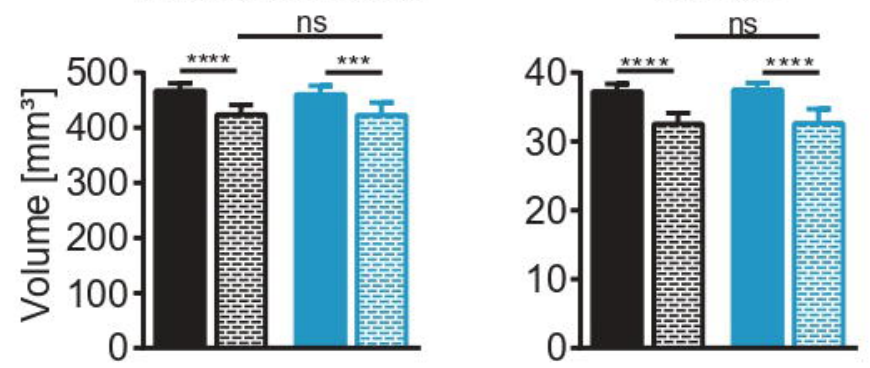

Copula pyramidis
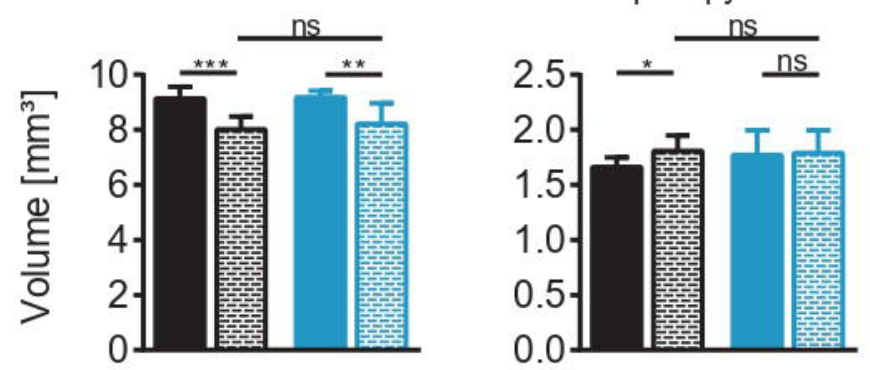

Piriform area, molecular layer

Intermediodorsal nucleus

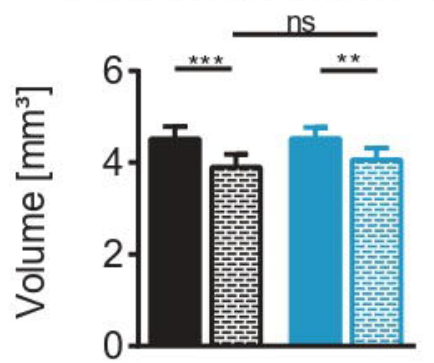
of the thalamus

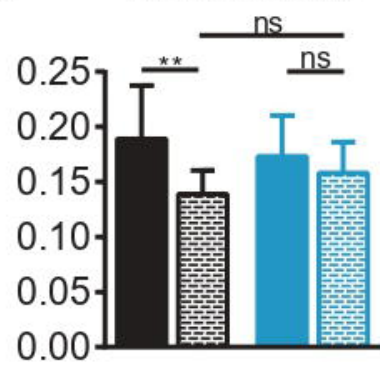


Figure S4

bioRaiv preprint doi: https://doi.org/10.1101/2020.10.13.337154; this version posted October 13, 2020. The copyright holder for this preprint (which was not certified by peer review) is the author/funder. All rights reserved. No reuse allowed without permission.

WT-vehicle

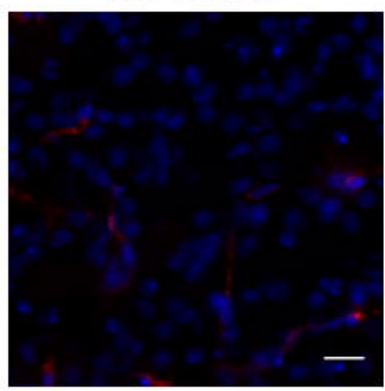

R6/1-vehicle
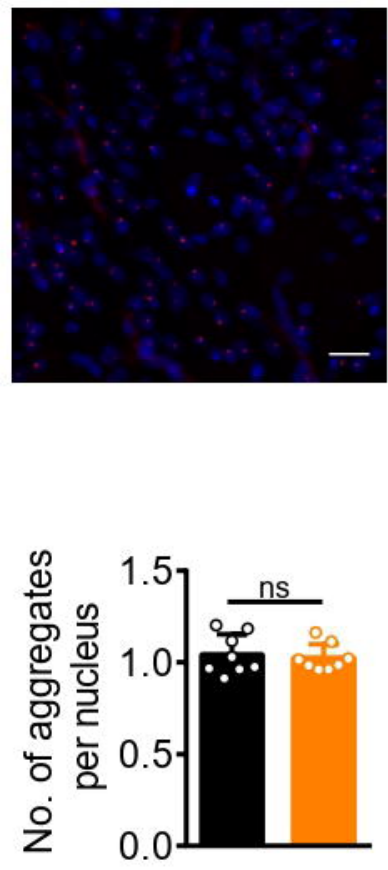

R6/1-RGFP109

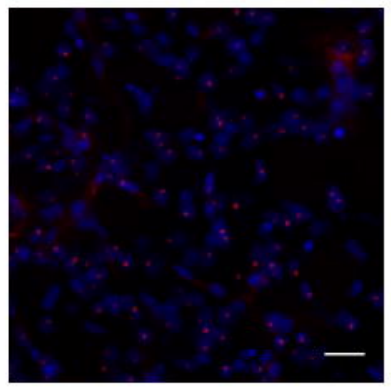

R6/1 - vehicle

R6/1 - RGFP109

B

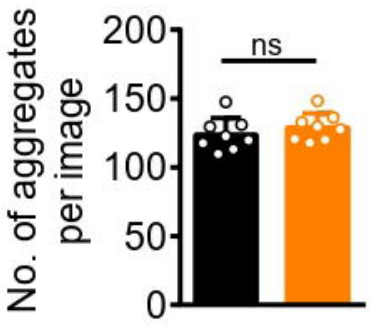

WT - vehicle \# R6/1 - vehicle WT - RGFP109 \# \#/1 - RGFP109
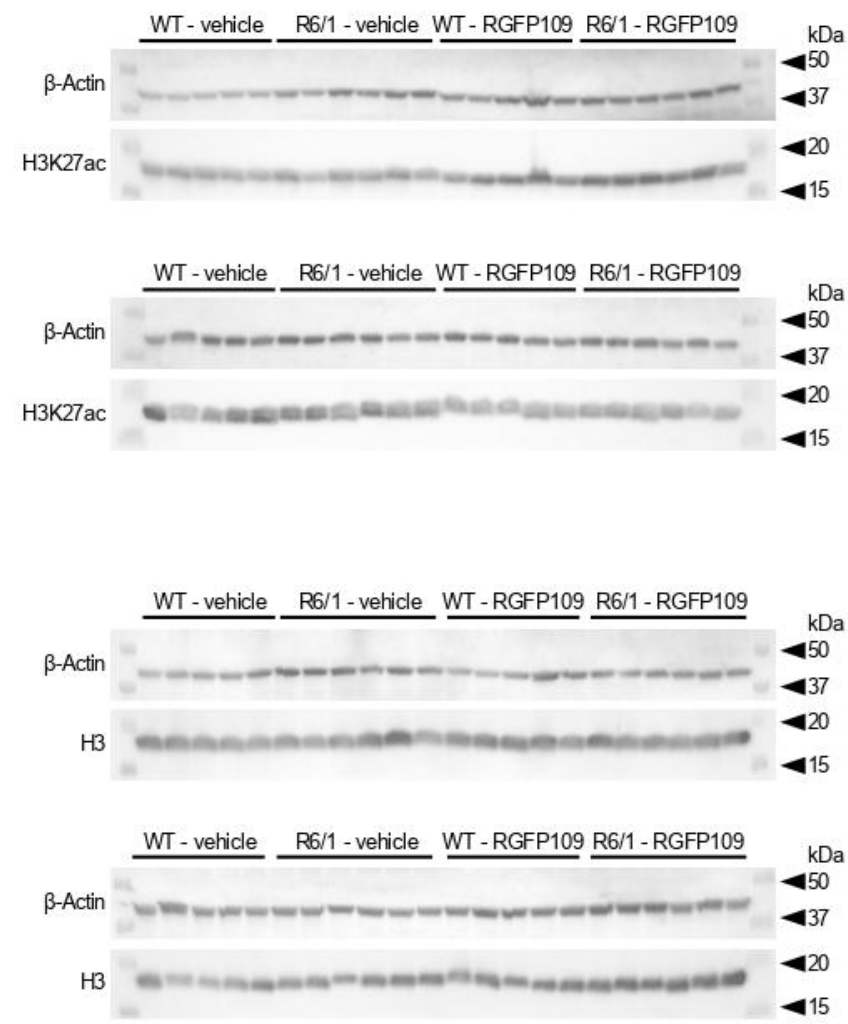
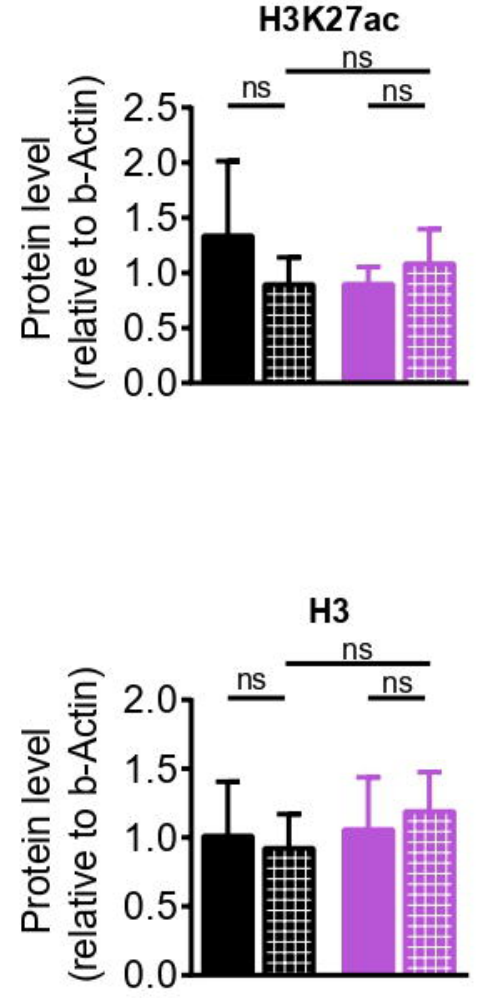